\title{
HODGE GENERA OF ALGEBRAIC VARIETIES, II.
}

\author{
SYLVAIN E. CAPPELL, ANATOLY LIBGOBER, LAURENTIU MAXIM, AND JULIUS L. SHANESON
}

\begin{abstract}
We study the behavior of Hodge-theoretic genera under morphisms of complex algebraic varieties. We prove that the additive $\chi_{y}$-genus which arises in the motivic context satisfies the so-called "stratified multiplicative property", which shows how to compute the invariant of the source of a proper surjective morphism from its values on various varieties that arise from the singularities of the map. By considering morphisms to a curve, we obtain a Hodge-theoretic analogue of the Riemann-Hurwitz formula. We also consider the contribution of monodromy to the $\chi_{y}$-genus of a smooth projective family, and prove an Atiyah-Meyer formula for twisted $\chi_{y}$-genera. This formula measures the deviation from multiplicativity of the $\chi_{y}$-genus, and expresses the correction terms as higher-genera associated to cohomology classes of the quotient of the total period domain by the action of the monodromy group. By making use of Saito's theory of mixed Hodge modules, we also obtain characteristic class formulae of Atiyah-Meyer type. In the last section we use intersection homology for the study of twisted Hodge-theoretic genera in the singular setting: we extend some results of [10] regarding the stratified multiplicative property of the $I \chi_{y}$-genus, and conjecture a Atiyah-Meyer type formula for twisted $I \chi_{y}$-genera.
\end{abstract}

\section{Contents}

1. Introduction

2. Hodge genera and singularities of maps

2.1. Hodge genera. Definitions.

2.2. Basics of Saito's theory of mixed Hodge modules.

2.3. Spectral sequences of mixed Hodge modules.

2.4. Multiplicativity properties of $\chi_{y}$-genera.

2.5. $\quad \chi_{y}^{c}$-genera and singularities of maps.

2.6. $\chi_{y}$-genera and mixed Hodge modules.

3. A Hodge-theoretic analogue of the Riemann-Hurwitz formula 15

3.1. Vanishing and nearby cycles.

3.2. A Riemann-Hurwitz formula for $\chi_{y}$-genera. 17

4. The presence of monodromy. An Atiyah-Meyer formula for the $\chi_{y}$-genus. 19

4.1. Hirzebruch classes of complex projective manifolds and the Hirzebruch-

Riemann-Roch theorem.

4.2. $\chi_{y}$-genera of smooth projective families.

Date: December 1, 2018.

2000 Mathematics Subject Classification. Primary 57R20, 32S20, 14C30, 32S35, 32S50, 14D05, 14D06, 14D07, 55N33; Secondary 57R45, 32S60, 13D15, 16E20. 
4.3. Higher $\chi_{y}$-genera and period domains.

5. Atiyah-Meyer type characteristic class formulae.

6. Twisted $\chi_{y}$-genera and characteristic classes in the singular setting. 32

6.1. Hodge intersection homology genera and the stratified multiplicative property. 32

6.2. Conjectural formulae for twisted intersection homology genera.

References

\section{INTRODUCTION}

In the mid 1950's, Chern, Hirzebruch and Serre [14 proved that if $F \rightarrow E \rightarrow B$ is a fiber bundle of closed oriented topological manifolds such that the fundamental group of $B$ acts trivially on the cohomology of $F$, then the signatures of the spaces involved are related by the following multiplicative relation: $\sigma(E)=\sigma(F) \sigma(B)$. A decade later, Atiyah [2, and respectively Kodaira [30, observed that without the assumption on the action of the fundamental group the multiplicativity relation fails. Moreover, Atiyah showed that the deviation from multiplicativity is controlled by the cohomology of the fundamental group of $B$.

The goal of this paper is to describe in a systematic way multiplicativity properties of the Hirzebruch $\chi_{y}$-genus (and the associate characteristic classes) for fibrations of algebraic manifolds. We extend the results of Chern, Hirzebruch and Serre in several different directions. First, we show that in the case when the Chern-Hirzebruch-Serre assumption of the triviality of the monodromy action is fulfilled, the $\chi_{y}$-genus is multiplicative. Since by the Hodge index theorem, the signature of a Kähler manifold is one of the values of the $\chi_{y}$-genus, this theorem can be viewed as an extension of the Chern-Hirzebruch-Serre result in the algebraic case. Secondly, we consider fibrations with non-trivial monodromy action. We derive a formula for the $\chi_{y}$-genus of $E$ in which the correction from the multiplicativity of the $\chi_{y}$-genus is measured via pullbacks under the period map associated with our fibration of certain cohomology classes of the quotient of the period domain by the action of the monodromy group. For some manifolds $F$ serving as a fiber of the fibration in discussion, as well as in the case when one is interested in the value of polynomial $\chi_{y}$ yielding the signature, the quotient of the period domain is the classifying space of the monodromy group and our correction terms coincide with those of Atiyah. In fact, the Atiyah terms are the appropriate Novikov type higher-signatures, and our correction terms are extensions of these Novikov invariants to the algebraic category. We indicate that recently established birational properties of higher genera [6, 7, 35] are valid also for "the corrections to multiplicativity" that we introduce here. Analogous characteristic class formulae are also discussed.

We now discuss in more detail the content of each section and summarize our main results.

In Section 2, we study the behavior of $\chi_{y}$-genera under maps of complex algebraic varieties. We first consider a morphism $f: E \rightarrow B$ of complex algebraic varieties with $B$ smooth 
and connected, which is a fiber bundle in the (strong) complex topology, and show that under certain assumptions on monodromy the $\chi_{y}$-genera are multiplicative (cf. Lemma 2.6 and Lemma 2.7). Such multiplicativity properties of genera were previously studied in certain special cases in connection with rigidity (e.g., see [27, 33]). For instance, Hirzebruch's $\chi_{y}$-genus is multiplicative in bundles of (stably) almost complex manifolds with structure group a compact connected Lie group (the latter condition implies trivial monodromy), and in fact it is uniquely characterized by this property. The proof of our multiplicativity result uses the fact that the Leray spectral sequences of the map $f$ are spectral sequences in the category of mixed Hodge structures. The latter claim is a consequence of Saito's theory of mixed Hodge modules (e.g., see [37]), and is discussed in some detail in $\$ 2.3$.

In Section 2.5, we consider proper morphisms that are allowed to have singularities, and extend the above multiplicativity property to the stratified case. More precisely, we prove that, under the assumption of trivial monodromy along the strata of our map, the additive $\chi_{y}$-genus that arises in the motivic context satisfies the so-called "stratified multiplicative property" (cf. Proposition 2.11 and Corollary 2.13). This property shows how to compute the invariant of the source of a proper surjective morphism from its values on various varieties that arise from the singularities of the map, thus yielding powerful topological constraints on the singularities of any algebraic map. It also provides a method of inductively computing these genera of varieties. A similar result was obtained by Cappell, Maxim and Shaneson, for the behavior of intersection homology Hodge-theoretic invariants, both genera and characteristic classes (see [10], and also [9]). Such formulae were first predicted by Cappell and Shaneson in the early 1990's, see the announcements [12, 43], following their earlier work on stratified multiplicative properties for signatures and associated topological characteristic classes defined using intersection homology [11].

In the special case of maps to a smooth curve, and under certain assumptions for the monodromy along the strata of special fibers, in $\$ 3.2$ we obtain a Hodge-theoretic analogue of the Riemann-Hurwitz formula [29]. The proof uses Hodge-theoretic aspects of the nearby and vanishing cycles in the context of one-parameter degenerations of projective varieties.

The contribution of monodromy to $\chi_{y}$-genera is studied in Section 4 . This can be applied to compute the summands arising from singularities in the above formulae. We consider a smooth proper map $f: E \rightarrow B$ of smooth projective varieties (thus a fibration in the strong topology), and compute $\chi_{y}(E)$ so that the (monodromy) action of $\pi_{1}(B)$ on the cohomology of the typical fiber is taken into account (see Theorem 4.1). The proof uses the Hirzebruch-Riemann-Roch theorem [26] and standard facts from the classical Hodge theory. Our formula (4.2) is a Hodge-theoretic analogue of Atiyah's formula for the signature of fiber bundles [2], and measures the deviation from multiplicativity of the $\chi_{y}$-genus in the presence of monodromy. In Section 4.3, this deviation is expressed in terms of higher-genera associated to cohomology classes of the quotient of the total period domain by the action of the monodromy group. As a corollary of our formula (4.2), we point out that if the action of $\pi_{1}(B)$ on the cohomology of the typical fiber $F$ preserves the Hodge filtration, then the $\chi_{y}$-genus is still multiplicative, i.e., $\chi_{y}(E)=\chi_{y}(B) \cdot \chi_{y}(F)$. This assertion is false in the non-compact case (see Example 2.9 (2)). As a byproduct of the proof of Theorem 
4.1, we obtain a Hodge-theoretic analogue of Meyer's formula for twisted signatures [31] (see Corollary 4.6).

In Section 5, we extend some of the above mentioned results on $\chi_{y}$-genera to AtiyahMeyer type formulae for the corresponding Hirzebruch characteristic classes. The proofs are much more involved, and use in an essential way the construction of Hirzebruch classes via Saito's theory of mixed Hodge modules (cf. [8]).

In the last section we discuss possible extensions of the monodromy formulae (4.2) and (4.7) to the singular setting. This is motivated by the considerations in [10, and by an extension of the Atiyah-Meyer signature formula to the singular case, which is due to Banagl, Cappell and Shaneson (cf. [3]). One possible extension to the singular setting makes use of intersection homology. In $\$ 6.1$, we show how twisted Hodge-theoretic intersection homology genera arise naturally in geometric mapping situations (the case of twisted signatures was considered in [11]), and we extend some results of [10] concerning the stratified multiplicative property for the $I \chi_{y}$-genus. By analogy with the work [3] on twisted signatures of strongly transverse Poincaré local systems, in $\$ 6.2$ we conjecture an extension of the monodromy formula (4.7) to the singular setting, and indicate an outline of its proof. We provide evidence for our conjecture by proving it in a special case, namely for projective varieties with a zero-dimensional singular locus.

It is conceivable that many of our results remain valid in a more general context (e.g., for complete algebraic varieties or even compactifiable complex analytic varieties). However, for simplicity, we chose to present our Atiyah-Meyer type results in the projective algebraic setting.

We have tried to make this paper as self-contained as possible. For this reason, in $₫ 2.2$ we provide necessary background on Saito's theory of mixed Hodge modules, and in 93.1 we recall Deligne's formalism of nearby and vanishing cycles. However, we assume reader's familiarity with certain aspects of Deligne's Hodge theory ([18, 34]).

Acknowledgements. We are grateful to Jörg Schürmann for reading a first draft of this work, and for making many valuable comments and suggestions for improvement. We also thank Mark Andrea de Cataldo, Alexandru Dimca and Nero Budur for many inspiring conversations on this subject.

\section{Hodge GENERA AND SINGUlarities OF MAPS}

2.1. Hodge genera. Definitions. In this section, we define the Hodge-theoretic invariants of complex algebraic varieties, which will be studied in the sequel. We assume reader's familiarity with Deligne's theory of mixed Hodge structures [18].

For any complex algebraic variety $Z$, we define the $\chi_{y}^{c}$-genus in terms of the Hodge-Deligne numbers of compactly supported cohomology of $Z$ (cf. [17]). More precisely,

$$
\chi_{y}^{c}(Z)=\sum_{p}\left(\sum_{i, q}(-1)^{i-p} h^{p, q}\left(H_{c}^{i}(Z ; \mathbb{C})\right)\right) y^{p}=\sum_{i, p \geq 0}(-1)^{i-p} \operatorname{dim}_{\mathbb{C}} G r_{F}^{p} H_{c}^{i}(Z, \mathbb{C}) \cdot y^{p}
$$


where $h^{p, q}\left(H_{c}^{i}(Z ; \mathbb{C})\right)=\operatorname{dim}_{\mathbb{C}} G r_{F}^{p}\left(G r_{p+q}^{W} H_{c}^{i}(Z) \otimes \mathbb{C}\right)$, with $F^{\bullet}$ and $W \bullet$ the Hodge and respectively the weight filtration of Deligne's mixed Hodge structure on $H_{c}^{i}(Z)$. Similarly, we define the $\chi_{y}$-genus of $Z, \chi_{y}(Z)$, by using the Hodge-Deligne numbers of $H^{*}(Z ; \mathbb{C})$. If $Z$ is smooth and projective, then each cohomology group $H_{c}^{i}(Z ; \mathbb{C})=H^{i}(Z ; \mathbb{C})$ has a pure Hodge structure of weight $i$, and the above formulae define Hirzebruch's $\chi_{y}$-genus (cf. [26]). Note that for any complex variety $Z$, we have that $\chi_{-1}^{c}(Z)=\chi_{-1}(Z)=\chi(Z)$ is the usual Euler characteristic, where for the first equality we refer to [22], pp. 141-142. Similarly, $\chi_{0}$ and $\chi_{0}^{c}$ are two possible extensions to singular varieties of the arithmetic genus.

The compactly supported $\chi_{y}$-genus, $\chi_{y}^{c}$, satisfies the so-called "scissor relations" for complex varieties, that is: $\chi_{y}^{c}(Z)=\chi_{y}^{c}(W)+\chi_{y}^{c}(Z \backslash W)$, for $W$ a closed subvariety of $Z$. Therefore, $\chi_{y}^{c}$ can be defined on $K_{0}\left(\operatorname{Var}_{\mathbb{C}}\right)$, the Grothendieck group of varieties over $\mathbb{C}$ which arises in the motivic context.

More generally, we can define $\chi_{y^{-}}$-genera on the Grothendieck group of mixed Hodge structures $K_{0}(m h s)=K_{0}\left(D^{b} m h s\right)$, where we denote by $m h s$ the abelian category of (rational) mixed Hodge structures. Indeed, if $K \in m h s$, define

$$
\chi_{y}([K]):=\sum_{p} \operatorname{dim}_{\mathbb{C}} G r_{F}^{p}(K \otimes \mathbb{C}) \cdot(-y)^{p},
$$

where $[K]$ is the class of $K$ in $K_{0}(m h s)$. This is well-defined on $K_{0}(m h s)$ since the functor $G r_{F}^{p}$ preserves exactness. For $K^{\bullet}$ a bounded complex of mixed Hodge structures, we define

$$
\left[K^{\bullet}\right]:=\sum_{i \in \mathbb{Z}}(-1)^{i}\left[K^{i}\right] \quad \in K_{0}(m h s)
$$

and note that we have:

$$
\left[K^{\bullet}\right]=\sum_{i \in \mathbb{Z}}(-1)^{i}\left[H^{i}\left(K^{\bullet}\right)\right]
$$

In view of (2.1), we set

$$
\chi_{y}\left(\left[K^{\bullet}\right]\right):=\sum_{i \in \mathbb{Z}}(-1)^{i} \chi_{y}\left(\left[K^{i}\right]\right) .
$$

In this language, we have that:

$$
\chi_{y}^{c}(Z)=\chi_{y}\left(\left[H_{c}^{\bullet}(Z ; \mathbb{Q})\right]\right)
$$

and

$$
\chi_{y}(Z)=\chi_{y}\left(\left[H^{\bullet}(Z ; \mathbb{Q})\right]\right),
$$

where $H_{c}^{\bullet}(Z ; \mathbb{Q})$ and $H^{\bullet}(Z ; \mathbb{Q})$ are regarded as bounded complexes of mixed Hodge structures, with all differentials equal to zero.

2.2. Basics of Saito's theory of mixed Hodge modules. Even though the theory of mixed Hodge modules is very involved, in this section we give a brief overview adapted to our needs, and we show some quick applications in the following sections. The standard references for the algebraic case are Saito's papers [38] and [37], §4, but see also the book 34, Chapter 14, for a brief survey. 
We recall that for any complex algebraic variety $Z$, the derived category of bounded cohomologically constructible complexes of sheaves of $\mathbb{Q}$-vector spaces on $Z$ is denoted by $D_{c}^{b}(Z)$, and it contains as a full subcategory the category $\operatorname{Perv}_{\mathbb{Q}}(Z)$ of perverse $\mathbb{Q}$-complexes. The Verdier duality operator $\mathbb{D}_{Z}$ is an involution on $D_{c}^{b}(Z)$ preserving $\operatorname{Perv}_{\mathbb{Q}}(Z)$. Associated to a morphism $f: X \rightarrow Y$ of complex algebraic varieties, there are pairs of adjoint functors $\left(f^{*}, R f_{*}\right)$ and $\left(f^{!}, R f_{!}\right)$between the respective categories of cohomologically constructible complexes, which are interchanged by Verdier duality. For details, see the books [21, 40].

M. Saito associated to a complex algebraic variety $Z$ an abelian category $M H M(Z)$, the category of mixed Hodge modules on $Z$, together with a faithful forgetful functor

$$
\text { rat }: D^{b} M H M(Z) \rightarrow D_{c}^{b}(Z)
$$

such that $\operatorname{rat}(M H M(Z)) \subset \operatorname{Perv}_{\mathbb{Q}}(Z)$. For $M^{\bullet} \in D^{b} M H M(Z), \operatorname{rat}\left(M^{\bullet}\right)$ is called the underlying rational complex of $M^{\bullet}$.

We say that $M \in M H M(Z)$ is supported on $S$ if and only if $\operatorname{rat}(M)$ is supported on $S$. Saito showed that the category of mixed Hodge modules supported on a point coincides with the category of (graded) polarizable rational mixed Hodge structures. In this case, the functor rat associates to a mixed Hodge structure the underlying rational vector space.

Since $M H M(Z)$ is an abelian category, the cohomology groups of any complex $M^{\bullet} \in$ $D^{b} M H M(Z)$ are mixed Hodge modules. The underlying rational complexes of the cohomology groups of a complex of mixed Hodge modules are the perverse cohomologies of the underlying rational complex, that is, $\operatorname{rat}\left(H^{j}\left(M^{\bullet}\right)\right)={ }^{p} \mathcal{H}^{j}\left(\operatorname{rat}\left(M^{\bullet}\right)\right)$.

The Verdier duality functor $\mathbb{D}_{Z}$ lifts to $M H M(Z)$ as an involution, in the sense that it commutes with the forgetful functor: rat $\circ \mathbb{D}_{Z}=\mathbb{D}_{Z} \circ$ rat.

For a morphism $f: X \rightarrow Y$ of complex algebraic varieties, there are induced functors $f_{*}, f_{!}: D^{b} M H M(X) \rightarrow D^{b} M H M(Y)$ and $f^{*}, f^{!}: D^{b} M H M(Y) \rightarrow D^{b} M H M(X)$, exchanged under the Verdier duality functor, and which lift the analogous functors on the level of constructible complexes. Moreover, if $f$ is proper, then $f_{!}=f_{*}$.

Let us give a rough picture of what the objects in Saito's category of mixed Hodge modules look like. For $Z$ smooth, $M H M(Z)$ is a full subcategory of the category of objects $((M, F), K, W)$ such that:

(1) $(M, F)$ is an algebraic holonomic filtered $\mathcal{D}$-module $M$ on $Z$, with an increasing "Hodge" filtration $F$ by coherent algebraic $\mathcal{O}_{Z}$-modules;

(2) $K \in \operatorname{Perv}_{\mathbb{Q}}(Z)$ is the underlying rational sheaf complex, and there is a quasiisomorphism $\alpha: D R(M) \simeq \mathbb{C} \otimes K$ in $\operatorname{Perv}_{\mathbb{C}}(Z)$, where $D R$ is the de Rham functor shifted by the dimension of $Z$;

(3) $W$ is a pair of (weight) filtrations on $M$ and $K$ compatible with $\alpha$.

For a singular variety $Z$, one works with local embeddings into manifolds and corresponding filtered $\mathcal{D}$-modules with support on $Z$. In this notation, the functor rat is defined by $\operatorname{rat}((M, F), K, W)=K$.

A complex $M^{\bullet} \in D^{b} M H M(Z)$ is mixed of weight $\leq k$ (resp. $\geq k$ ) if $G r_{i}^{W} H^{j} M^{\bullet}=0$ for all $i>j+k$ (resp. $i<j+k$ ), and it is pure of weight $k$ if $G r_{i}^{W} H^{j} M^{\bullet}=0$ for all $i \neq j+k$. If $f$ is a map of algebraic varieties, then $f_{!}$and $f^{*}$ preserve weight $\leq k$, and 
$f_{*}$ and $f^{!}$preserve weight $\geq k$. If $M^{\bullet} \in D^{b} M H M(Z)$ is of weight $\leq k$ (resp. $\geq k$ ), then $H^{j} M^{\bullet}$ has weight $\leq j+k$ (resp. $\geq j+k$ ).

If $j: U \hookrightarrow Z$ is a Zariski-open subset in $Z$, then the intermediate extension $j_{!_{*}}$ (cf. [5]) preserves the weights.

Following 37, there exists a unique object $\mathbb{Q}^{H} \in M H M($ point $)$ such that $\operatorname{rat}\left(\mathbb{Q}^{H}\right)=\mathbb{Q}$ and $\mathbb{Q}^{H}$ is of type $(0,0)$. In fact, $\mathbb{Q}^{H}=((\mathbb{C}, F), \mathbb{Q}, W)$, with $g r_{i}^{F}=0=g r_{i}^{W}$ for all $i \neq 0$, and $\alpha: \mathbb{C} \rightarrow \mathbb{C} \otimes \mathbb{Q}$ the obvious isomorphism. For a complex variety $Z$, define $\mathbb{Q}_{Z}^{H}:=a_{Z}^{*} \mathbb{Q}^{H} \in D^{b} M H M(Z)$ with $a_{Z}: Z \rightarrow$ point the map to a point. If $Z$ is smooth of dimension $n$, then $\mathbb{Q}_{Z}[n] \in \operatorname{Perv}\left(\mathbb{Q}_{X}\right)$ and $\mathbb{Q}_{Z}^{H}[n] \in M H M(Z)$ is a single mixed Hodge module (in degree 0), explicitely described by $\mathbb{Q}_{Z}^{H}[n]=\left(\left(\mathcal{O}_{Z}, F\right), \mathbb{Q}_{Z}[n], W\right)$, where $F$ and $W$ are trivial filtrations so that $g r_{i}^{F}=0=g r_{i+n}^{W}$ for all $i \neq 0$. So if $Z$ is smooth of dimension $n$, then $\mathbb{Q}_{Z}^{H}[n]$ is pure of weight $n$. By the stability of the intermediate extension functor, this shows that if $Z$ is any algebraic variety and $j: U \hookrightarrow Z$ is the inclusion of a smooth Zariski-open subset, then the intersection cohomology module $I C_{Z}^{H}:=j_{! *}\left(\mathbb{Q}_{U}^{H}[n]\right)$ is pure of weight $n$.

More generally, if $\mathbb{V}$ is a polarized variation of Hodge structures of weight $k$ with qusiunipotent monodromy at infinity defined on a Zariski-open subset $U$ of $Z$, then $\mathbb{V}$ corresponds to a smooth mixed Hodge module $\mathbb{V}^{H}[n]$ on $U$ (i.e., the associated rational complex is a local system) of weight $k+n$, whose underlying perverse sheaf is $\mathbb{V}[n]$ (see [36], Thm. 5.4.3, [38], §2, 34], Thm. 14.30). So the twisted (middle-perversity) intersection homology complex $I C_{Z}^{H}(\mathbb{V}):=j_{! *}\left(\mathbb{V}^{H}[n]\right)$ is a mixed Hodge module, pure of weight $k+n$.

The following result is implicit in the work of Saito.

Proposition 2.1. Let $Z$ be a n-dimensional irreducible projective variety, and $\mathbb{V}$ a polarized variation of Hodge structures of weight $k$ defined on a Zariski-open dense subset of $Z$. Then the intersection cohomology group $I H^{j}(Z ; \mathbb{V})$ carries a pure Hodge structure of weight $j+k$.

Proof. Let $a_{Z}: Z \rightarrow$ point be the constant map to a point. Since $a_{Z}$ is proper, $a_{Z *}$ preserves the weights. The claim follows from the isomorphism

$$
I H^{j}(Z ; \mathbb{V}) \cong \mathbb{H}^{j-n}\left(Z ; I C_{Z}^{H}(\mathbb{V})\right) \cong H^{j-n}\left(a_{Z *} I C_{Z}^{H}(\mathbb{V})\right),
$$

by noting that $a_{Z *} I C_{Z}^{H}(\mathbb{V})$ is a pure complex of weight $n+k$, thus $H^{j-n}\left(a_{Z *} I C_{Z}^{H}(\mathbb{V})\right)$ is a pure Hodge module of weight $j+k$ supported over a point. Since over a point mixed Hodge modules are exactly the (graded) polarizable mixed Hodge structures, the latter is a Hodge structure of weight $j+k$.

The following corollary will be needed in the sequel:

Corollary 2.2. Let $Z$ be a smooth complex projective algebraic variety, and $\mathbb{V}$ a polarized variation of Hodge structures of weight $k$ defined on $Z$. Then the cohomology group $H^{j}(Z ; \mathbb{V})$ carries a pure Hodge structure of weight $j+k$.

\footnotetext{
${ }^{1}$ This condition is automatically satisfied is $\mathbb{V}$ is a variation of $\mathbb{Z}$-Hodge structures, by the monodromy theorem, 34, Thm. 11.8.
} 
Remark 2.3. More generally, if the variety $Z$ in Proposition 2.1 and Corollary 2.2 is not necessarily projective, and if $\mathbb{V}$ is a polarized variation of Hodge structures or, more generally, an admissible variation of mixed Hodge structures (for a definition, see [34], Def. 14.47 and the references therein) on (a Zariski-open dense subset of) $Z$, then the associated (intersection) cohomology groups carry natural mixed Hodge structures.

2.3. Spectral sequences of mixed Hodge modules. In this section, we justify the claim that certain Leray-type spectral sequences (e.g., the Leray spectral sequence of an algebraic morphism, or the hypercohomology spectral sequence) are in fact spectral sequences of mixed Hodge structures.

From the general theory of spectral sequences, since the category of mixed Hodge modules is abelian, the canonical filtration $\tau$ on $D^{b} M H M(Z)$ preserves complexes of mixed Hodge modules. Therefore, the second fundamental spectral sequence ([34], §A.3.4) for any (left exact) functor $F$ sending mixed Hodge modules to mixed Hodge modules, that is, the spectral sequence

$$
E_{2}^{p, q}=H^{p} F\left(H^{q}\left(M^{\bullet}\right)\right) \Longrightarrow H^{p+q} F\left(M^{\bullet}\right),
$$

is a spectral sequences of mixed Hodge modules.

Note that the canonical $t$-structure $\tau$ on $D^{b} M H M(Z)$ corresponds to the perverse truncation ${ }^{p} \tau$ on $D_{c}^{b}(Z)$. However, Saito ([37], Remark 4.6(2)) constructed another $t$-structure ${ }^{\prime} \tau$ on $D^{b} M H M(Z)$ that corresponds to the classical $t$-structure on $D_{c}^{b}(Z)$. By using the $t$-structure ${ }^{\prime} \tau$ in the construction of the second fundamental spectral sequence above, one can show that the classical Leray spectral sequences are in fact spectral sequences of mixed Hodge structures.

Example 2.4. Leray spectral sequences.

(1) Let $Z$ be a complex algebraic variety. Then for $\mathcal{F}^{\bullet}$ a bounded complex of sheaves with constructible cohomology on $Z$, we have the spectral sequence with the $E_{2}$-term given by

$$
E_{2}^{p, q}=H^{p}\left(Z ; \mathcal{H}^{q}\left(\mathcal{F}^{\bullet}\right)\right) \Longrightarrow \mathbb{H}^{p+q}\left(Z ; \mathcal{F}^{\bullet}\right) .
$$

This spectral sequence is induced by the natural filtration on the complex $\mathcal{F}^{\bullet}$, and if $\mathcal{F}^{\bullet}$ underlies a complex of mixed Hodge modules, then the spectral sequence is compatible with mixed Hodge structures: this follows by using the $t$-structure ' $\tau$ defined in [37], Remark 4.6(2), and the fundamental spectral sequence (2.3) for $F=\Gamma(Z, \cdot)=\left(a_{Z}\right)_{*}$, together with the fact that mixed Hodge modules over a point are (graded polarizable) mixed Hodge structures.

Similarly, by taking $F=\Gamma_{c}(Z, \cdot)=\left(a_{Z}\right)$ ! together with the $t$-structure ' $\tau$ above, the compactly supported hypercohomology Leray spectral sequence

$$
E_{2}^{p, q}=H_{c}^{p}\left(Z ; \mathcal{H}^{q}\left(\mathcal{F}^{\bullet}\right)\right) \Longrightarrow \mathbb{H}_{c}^{p+q}\left(Z ; \mathcal{F}^{\bullet}\right) .
$$

is a spectral sequence in the category of mixed Hodge structures, provided $\mathcal{F}^{\bullet}$ underlies a bounded complex of mixed Hodge modules. 
(2) Let $f: E \rightarrow B$ be a morphism of complex algebraic varieties. The Leray spectral sequence for $f$

$$
E_{2}^{p, q}=H^{p}\left(B, R^{q} f_{*} \mathbb{Q}_{E}\right) \Longrightarrow H^{p+q}(E)
$$

is a spectral sequence of mixed Hodge structures: this follows by using ' $\tau$ on $D^{b} M H M(B)$, and the example above applied to $\mathcal{F}^{\bullet}=R f_{*} \mathbb{Q}_{E}$, that underlies $M^{\bullet}=f_{*} \mathbb{Q}_{E}^{H} \in D^{b} M H M(B)$. Similarly, there is a compactly supported version of the Leray spectral sequence for $f$ in the category of mixed Hodge structures, namely

$$
E_{2}^{p, q}=H_{c}^{p}\left(B, R^{q} f_{!} \mathbb{Q}_{E}\right) \Longrightarrow H_{c}^{p+q}(E) .
$$

By using the usual $t$-structure on $D^{b} M H M(B)$, hence the perverse $t$-structure on $D_{c}^{b}(B)$, we obtain that the perverse Leray spectral sequence for $f$,

$$
E_{2}^{i, j}=\mathbb{H}^{i}\left(B,{ }^{p} \mathcal{H}^{j}\left(f_{*} \mathcal{F}^{\bullet}\right)\right) \Longrightarrow \mathbb{H}^{i+j}\left(E ; \mathcal{F}^{\bullet}\right)
$$

is a spectral sequence in the category of mixed Hodge structures, provided $\mathcal{F}^{\bullet}$ underlies a bounded complex of mixed Hodge modules.

Remark 2.5. In the quasi-projective setting, a different proof of the fact that the spectral sequences (2.6) and (2.7) are spectral sequences of mixed Hodge structures was given by Arapura in [1].

2.4. Multiplicativity properties of $\chi_{y}$-genera. In this section we use the above spectral sequences in studying multiplicative properties of the Hodge-theoretic genera. We first prove the following:

Lemma 2.6. Let $E, B, F$ be complex algebraic varieties with $B$ smooth and connected, and let $p: E \rightarrow B$ be an algebraic morphism such that $p$ is locally trivial in the strong (complex) topology of $B$, with fiber $F$. Assume that the local systems $R^{j} p_{*} \mathbb{C}_{E}$ are constant for each $j$ (e.g., $\left.\pi_{1}(B)=0\right)$. Then

$$
\chi_{y}(E)=\chi_{y}(B) \chi_{y}(F) .
$$

If moreover, all $E, B$ and $F$ are smooth, then

$$
\chi_{y}^{c}(E)=\chi_{y}^{c}(B) \chi_{y}^{c}(F) .
$$

Proof. Consider the Leray spectral sequence of $p$, that is

$$
E_{2}^{p, q}=H^{p}\left(B, R^{q} p_{*} \mathbb{C}_{E}\right) \Longrightarrow H^{p+q}(E)
$$

In the category of algebraic varieties this is a spectral sequence of mixed Hodge structures (cf. §2.3).

Since the local systems $R^{q} p_{*} \mathbb{C}_{E}$ are constant, the corresponding variations of mixed Hodge structures are trivial as the natural morphism $H^{0}\left(B, R^{q} p_{*} \mathbb{C}_{E}\right) \rightarrow\left(R^{q} p_{*} \mathbb{C}_{E}\right)_{y}$ is a mixed Hodge structure isomorphism for any $y \in B$. Then we have $H^{p}\left(B, R^{q} p_{*} \mathbb{C}_{E}\right)=$ $H^{p}(B) \otimes\left(R^{q} p_{*} \mathbb{C}_{E}\right)_{y}$ as mixed Hodge structures, and there are mixed Hodge structure isomorphisms

$$
E_{2}^{p, q}=H^{p}(B) \otimes H^{q}(F)
$$


Since all differentials in the Leray spectral sequence are mixed Hodge structure morphisms, thus strict with respect to the Hodge and weight filtrations, by [18], Lemme 1.1.11 we get a spectral sequence for the Hodge components of a given type $(k, l)$ :

$$
E(k, l)_{2}^{p, q}:=G r_{F}^{k} G r_{k+l}^{W} E_{2}^{p, q} \Longrightarrow G r_{F}^{k} G r_{k+l}^{W} H^{p+q}(E)
$$

Now let $e^{k, l}$ be the Euler characteristic of Hodge-type $(k, l)$, i.e., for a complex algebraic variety $Z$ we define

$$
e^{k, l}(Z)=\sum_{i}(-1)^{i} h^{k, l}\left(H^{i}(Z)\right) .
$$

By the invariance of Euler characteristics under spectral sequences, from (2.9) and (2.10) we obtain

$$
\begin{aligned}
e^{k, l}(E) & =\sum_{i}(-1)^{i} \operatorname{dim}\left(\oplus_{p+q=i} E(k, l)_{2}^{p, q}\right) \\
& =\sum_{r+t=k, s+u=l} e^{r, s}(B) e^{t, u}(F) .
\end{aligned}
$$

The multiplicativity of $\chi_{y}$ follows by noting that for a variety $Z$,

$$
\chi_{y}(Z)=\sum_{k, l} e^{k, l}(Z) \cdot(-y)^{k} .
$$

The claim about the multiplicativity of the $\chi_{y}^{c}$-genus follows by Poincaré Duality, by noting that if $Z$ is a smooth complex algebraic variety of dimension $n$, then $\chi_{y}^{c}(Z)=(-y)^{n} \chi_{y^{-1}}(Z)$. Indeed, the Poincaré duality isomorphism takes classes of type $(p, q)$ in $H_{c}^{j}(Z)$ to classes of type $(n-p, n-q)$ in $H^{2 n-j}(Z)$, where $n=\operatorname{dim} Z$.

By noting that for any $b \in B,\left(R^{j} p_{!} \mathbb{C}_{E}\right)_{b} \cong H_{c}^{j}(F ; \mathbb{C})$, a similar argument applied to the compactly supported Leray spectral sequence (2.7) of the map $p$, yields the following

Lemma 2.7. Let $E, B, F$ be complex algebraic varieties with $B$ smooth and connected, and consider $p: E \rightarrow B$ an algebraic morphism such that $p$ is locally trivial in the strong (complex) topology of $B$ with fibre $F$. Assume that the local systems $R^{j} p_{!} \mathbb{C}_{E}$ are constant for each $j\left(\right.$ e.g., $\left.\pi_{1}(B)=0\right)$. Then

$$
\chi_{y}^{c}(E)=\chi_{y}^{c}(B) \chi_{y}^{c}(F) .
$$

Remark 2.8. The same argument can be used to show that the results of the above lemmas hold for the Hodge-Deligne polynomials (or the $E$-functions) defined by $E(Z ; u, v)=$ $\sum_{k, l} e^{k, l}(Z) u^{k} v^{l}$ (and similarly for the $E$-functions $E_{c}(Z ; u, v)$ defined by using the compactly supported cohomology). In particular, these results hold for the weight polynomials $W(Z ; t):=E(Z ; t, t)$ and respectively $W_{c}(Z ; t):=E_{c}(Z ; t, t)$ considered in [20]. In fact, Lemma 2.6] is modeled after [20], Theorem 6.1. 
Example 2.9. (1) As an example, consider the case of the Hopf fibration defining $\mathbb{C P}^{n}$. Then $\chi_{y}\left(\mathbb{C P}^{n}\right)=\chi_{y}^{c}\left(\mathbb{C P}^{n}\right)=1+(-y)+\cdots+(-y)^{n}, \chi_{y}^{c}\left(\mathbb{C}^{n+1} \backslash\{0\}\right)=(-y)^{n+1}-1$, $\chi_{y}^{c}\left(\mathbb{C}^{*}\right)=-y-1$, and by Poincaré Duality, $\chi_{y}\left(\mathbb{C}^{n+1} \backslash\{0\}\right)=1-(-y)^{n+1}, \chi_{y}\left(\mathbb{C}^{*}\right)=1+y$. Thus the multiplicativity for both $\chi_{y}$ and $\chi_{y}^{c}$ holds.

(2) Let $p$ be the Milnor fibration of a weighted homogeneous isolated hypersurface singularity at the origin in $\mathbb{C}^{n+1}$, that is, $F=\{p=1\} \hookrightarrow E=\mathbb{C}^{n+1} \backslash\{p=0\} \rightarrow B=\mathbb{C}^{*}$, for $p$ a weighted homogeneous polynomial in $n+1$ variables, with an isolated singular point at the origin. In this case, the monodromy is an algebraic morphism of finite order, so it preserves the filtrations in $H^{*}(F ; \mathbb{C})$. In other words, each $R^{j} p_{*} \mathbb{Q}_{E}$ is a (non-constant) local system of mixed Hodge structures (note that this is not the case for general singularities). The mixed Hodge structure on $H^{*}(F ; \mathbb{Q})$ is known by work of Steenbrink [44]. It turns out that even in this special case, the $\chi_{y}$-genera are not multiplicative (but see Section $\$ 4$ for the compact case). Here is a concrete example: let $p(x, y)=x^{3}-y^{2}$ defining the cuspidal cubic in $\mathbb{C}^{2}$. Then, in the notations above, by [44] we obtain that $\chi_{y}(F)=2 y+1$, so by Poincaré Duality it follows that $\chi_{y}^{c}(F)=-y-2$. It also follows easily that $\chi_{y}^{c}(E)=y^{2}+y$, $\chi_{y}^{c}(B)=-y-1$, and $\chi_{y}(E)=1+y, \chi_{y}(B)=1+y$.

Remark 2.10. The assumption of trivial monodromy is closely related to, but different from the situation of "algebraic piecewise trivial" maps coming up in the motivic context (e.g., see [8]). For example, the formula in Lemma 2.7 is true (without any assumption on monodromy) for a Zariski locally trivial fibration of possibly singular complex algebraic varieties (see [17], Corollary 1.9, or [8], Example 3.3).

2.5. $\chi_{y}^{c}$-genera and singularities of maps. In this section, by analogy with the results of [9, 10], we discuss the behavior of the $\chi_{y}^{c}$-genus under proper morphisms of algebraic varieties, and show that $\chi_{y}^{c}$ satisfies the stratified multiplicative property in the sense of [12]. The result will be further refined in $\$ 3$, in the case of maps onto curves.

Let $f: X^{n} \rightarrow Y^{m}$ be a proper map of complex algebraic varieties of indicated dimensions. Such a map can be stratified with subvarieties as strata, i.e., there exist finite algebraic Whitney stratifications $\mathcal{X}$ of $X$ and $\mathcal{S}$ of $Y$, such that for any component $S$ of a stratum of $Y, f^{-1}(S)$ is a union of connected components of strata of $\mathcal{X}$, each of which is mapping submersively to $S$. This implies that $f_{\mid f^{-1}(S)}: f^{-1}(S) \rightarrow S$ is a locally trivial map of Whitney stratified spaces (see 24], §I.1.6). For simplicity, we will assume that $f$ is smooth over the dense open stratum (e.g., the latter is connected). We denote by $F$ the general fiber of $f$, and by $F_{S}$ the fiber of $f$ above the singular stratum $S \in \mathcal{S}$.

We can now prove the following formula, showing the deviation from multiplicativity of the $\chi_{y}^{c}$-genus in the case of a stratified map (compare with results in [9, 10], and see also $\$ 6.1$ below):

Proposition 2.11. Let $f: X \rightarrow Y$ be a proper surjective morphism of (possibly singular) complex algebraic varieties. Let $\mathcal{S}$ be the set of components of open strata of $Y$ in a stratification of $f$, and assume $\pi_{1}(S)=0$ for all $S \in \mathcal{S}$. For $S \in \mathcal{S}$, define $\hat{\chi}_{y}^{c}(\bar{S})$ inductively by 
the formula:

$$
\hat{\chi}_{y}^{c}(\bar{S})=\chi_{y}^{c}(\bar{S})-\sum_{W<S} \hat{\chi}_{y}^{c}(\bar{W}),
$$

where the sum is over all $W \in \mathcal{S}$ with $W \subset \bar{S} \backslash S$. Then:

$$
\chi_{y}^{c}(X)=\chi_{y}^{c}(Y) \chi_{y}^{c}(F)+\sum_{S \in \mathcal{S}, \operatorname{dim} S<\operatorname{dim} Y} \hat{\chi}_{y}^{c}(\bar{S}) \cdot\left[\chi_{y}^{c}\left(F_{S}\right)-\chi_{y}^{c}(F)\right] .
$$

Proof. Note that by the additivity of $\chi_{y}^{c}$-genera, we have that $\hat{\chi}_{y}^{c}(\bar{S})=\chi_{y}^{c}(S)$. Next, by additivity and multiplicativity for locally trivial fibrations with trivial monodromy (cf. Lemma 2.7), it is easy to see that:

$$
\chi_{y}^{c}(X)=\chi_{y}^{c}(Y) \chi_{y}^{c}(F)+\sum_{S \in \mathcal{S}, \operatorname{dim} S<\operatorname{dim} Y} \chi_{y}^{c}(S) \cdot\left[\chi_{y}^{c}\left(F_{S}\right)-\chi_{y}^{c}(F)\right] .
$$

The result follows.

\section{Example 2.12. Smooth blow-up}

Let $Y$ be a smooth subvariety of codimension $r+1$ in a smooth variety $X$. Let $\pi: \tilde{X} \rightarrow X$ be the blow-up of $X$ along $Y$. Then $\pi$ is an isomorphism over $X \backslash Y$ and a projective bundle (Zariski locally trivial) over $Y$, coresponding to the normal bundle of $Y$ in $X$ of rank $r+1$. The formula in Proposition 2.11 yields

$$
\chi_{y}^{c}(\tilde{X})=\chi_{y}^{c}(X)+\chi_{y}^{c}(Y) \cdot\left(-y+\cdots+(-y)^{r}\right) .
$$

Note that this formula also holds without any assumption on monodromy, by using instead Remark 2.10 and the fact that $\pi^{-1}(Y)$ is a Zariski locally trivial fibration over $Y$ with fiber $\mathbb{C P}^{r}$ (see [17], §1.10).

In the notations of Proposition 2.11, we also obtain the following fact which was claimed in [10]:

Corollary 2.13. Let $f: X \rightarrow Y$ be a proper surjective morphism of complex projective algebraic varieties, and assume $\pi_{1}(S)=0$ for all $S \in \mathcal{S}$. Then with the obvious definition for $\hat{\chi_{y}}(\bar{S})$, the following holds:

$$
\chi_{y}(X)=\chi_{y}(Y) \chi_{y}(F)+\sum_{S \in \mathcal{S}, \operatorname{dim} S<\operatorname{dim} Y} \hat{\chi_{y}}(\bar{S}) \cdot\left[\chi_{y}\left(F_{S}\right)-\chi_{y}(F)\right] .
$$

Remark 2.14. More generally, by Remark 2.8 and additivity, both formulae (2.11) and (2.13) above are satisfied by the Hodge-Deligne polynomial $E_{c}(-; u, v)$ defined by means of compactly supported cohomology. In other words, the polynomial $E_{c}(-; u, v)$ satisfies the stratified multiplicative property. 
2.6. $\chi_{y}$-genera and mixed Hodge modules. In this section, by using Saito's theory of mixed Hodge modules we derive some easy additivity properties of $\chi_{y}$-genera of complexes of mixed Hodge structures. We begin with a consequence of the fact that mixed Hodge modules over a point are just (graded-polarizable) mixed Hodge structures:

Lemma 2.15. Let $Z$ be a complex algebraic variety, and $a_{Z}: Z \rightarrow p t$ be the constant map to the point. For any bounded complex $M^{\bullet}$ of mixed Hodge modules on $Z$

$$
\mathbb{H}^{p}\left(Z ; M^{\bullet}\right):=H^{p}\left(\left(a_{Z}\right)_{*} M^{\bullet}\right) \quad \text { and } \quad \mathbb{H}_{c}^{p}\left(Z ; M^{\bullet}\right):=H^{p}\left(\left(a_{Z}\right) ! M^{\bullet}\right)
$$

are mixed Hodge structures. In particular, if $M$ is a mixed Hodge module on $Z$ whose underlying rational complex is the perverse sheaf $\mathcal{F}^{\bullet}$, then the hypercohomology group $\mathbb{H}^{p}\left(Z ; \mathcal{F}^{\bullet}\right)$ (and resp. $\mathbb{H}_{c}^{p}\left(Z ; \mathcal{F}^{\bullet}\right)$ ) is the rational vector space underlying $\mathbb{H}^{p}(Z ; M)$ (and resp. $\left.\mathbb{H}_{c}^{p}(Z ; M)\right)$, hence the former is a rational mixed Hodge structure.

As a corollary, we obtain some very useful facts for the global-to-local study of $\chi_{y}$-genera. If $Z$ is a complex algebraic variety, and $i: Y \hookrightarrow Z$ is a closed immersion, with $j: U \rightarrow Z$ the inclusion of the open complement, then there is a functorial distinguished triangle for $M^{\bullet} \in D^{b} M H M(Z)$, lifting the corresponding one from $D_{c}^{b}(Z)$ (cf [37], p. 321):

$$
j_{!} j^{*} M^{\bullet} \rightarrow M^{\bullet} \rightarrow i_{*} i^{*} M^{\bullet} \stackrel{[1]}{\rightarrow}
$$

In particular, by taking hypercohomology with compact supports in (2.14) and together with Lemma 2.15, we obtain that the following long exact sequence is a sequence in the category of mixed Hodge structures:

$$
\cdots \rightarrow \mathbb{H}_{c}^{p}\left(U ; j^{*} M^{\bullet}\right) \rightarrow \mathbb{H}_{c}^{p}\left(Z ; M^{\bullet}\right) \rightarrow \mathbb{H}_{c}^{p}\left(Y ; i^{*} M^{\bullet}\right) \rightarrow \cdots
$$

Therefore,

$$
\chi_{y}\left(\left[\mathbb{H}_{c}^{\bullet}\left(Z ; M^{\bullet}\right)\right]\right)=\chi_{y}\left(\left[\mathbb{H}_{c}^{\bullet}\left(Y ; M^{\bullet}\right)\right]\right)+\chi_{y}\left(\left[\mathbb{H}_{c}^{\bullet}\left(U ; M^{\bullet}\right)\right]\right) .
$$

As a corollary of (2.16), by induction on strata we obtain the following additivity property:

Corollary 2.16. Let $\mathcal{S}$ be the set of components of strata of an algebraic Whitney stratification of the complex algebraic variety $Z$. Then for any $M^{\bullet} \in D^{b} M H M(Z)$,

$$
\chi_{y}\left(\left[\mathbb{H}_{c}^{\bullet}\left(Z ; M^{\bullet}\right)\right]\right)=\sum_{S \in \mathcal{S}} \chi_{y}\left(\left[\mathbb{H}_{c}^{\bullet}\left(S ; M^{\bullet}\right)\right]\right) .
$$

Remark 2.17. By taking $M^{\bullet}=\mathbb{Q}_{Z}^{H}$ in (2.17), we obtain the usual additivity of the $\chi_{y}^{c}$ genus (recall that $\chi_{y}^{c}$ is defined on the Grothendieck group of complex varieties). This is a consequence of the fact that Deligne's and Saito's mixed Hodge structures on cohomology (with compact support) coincide. The latter assertion can be seen by construction if the variety can be embedded into a manifold, but in general it is a very deep result of Saito, see [39].

Each of the terms in the sum of the right-hand side of equation (2.17) can be further computed by means of the Leray spectral sequence for hypercohomology (cf. \$2.3). 
Let $Z$ be an algebraic variety, $M^{\bullet} \in D^{b} M H M(Z)$, and fix an algebraic Whitney stratification with respect to which $\mathcal{F}^{\bullet}:=\operatorname{rat}\left(M^{\bullet}\right) \in D_{c}^{b}(Z)$ has constructible cohomology. Let $\mathcal{S}$ be the set of components of strata, and fix $S \in \mathcal{S}$. Then $S$ is a non-singular complex algebraic variety. Moreover, each cohomology sheaf $\mathcal{H}^{q}\left(\mathcal{F}^{\bullet}\right)$ on $S$ is a local system underlying a variation of mixed Hodge structures. We first prove the following extension of Lemmas 2.6 and 2.7 (in fact, the latter follow from this for some distinguished choices of $M^{\bullet}$ ):

Proposition 2.18. Assume the local systems $\mathcal{H}^{j}\left(\mathcal{F}^{\bullet}\right)$ are constant on $S$ for each $j \in \mathbb{Z}$, e.g. $\pi_{1}(S)=0$. Then

$$
\chi_{y}\left(\left[\mathbb{H}^{\bullet}\left(S ; M^{\bullet}\right)\right]\right)=\chi_{y}(S) \cdot \chi_{y}\left(\left[\mathcal{H}^{\bullet}\left(\mathcal{F}^{\bullet}\right)_{x}\right]\right)
$$

and

$$
\chi_{y}\left(\left[\mathbb{H}_{c}^{\bullet}\left(S ; M^{\bullet}\right)\right]\right)=\chi_{y}^{c}(S) \cdot \chi_{y}\left(\left[\mathcal{H}^{\bullet}\left(\mathcal{F}^{\bullet}\right)_{x}\right]\right),
$$

where $\left[\mathcal{H}^{\bullet}\left(\mathcal{F}^{\bullet}\right)_{x}\right] \in K_{0}$ (mhs) is the complex (with all differentials zero) of mixed Hodge structures corresponding to stalk cohomologies of $\mathcal{F}^{\bullet}$ at a point in $S$.

Proof. This is a consequence of the fact that the spectral sequences (2.4) and (2.5), applied to the variety $S$ and to the complex $\left.\mathcal{F}^{\bullet}\right|_{S}$, are spectral sequences of mixed Hodge structures.

By our assumption, the variations of mixed Hodge structures $\mathcal{H}^{q}\left(\mathcal{F}^{\bullet}\right)$ on $S$ are trivial, hence by (2.4) and as in the proof of Lemma 2.6 there are mixed Hodge structure isomorphisms

$$
E_{2}^{p, q}=H^{p}(S) \otimes V^{q},
$$

where $V^{q}:=\mathcal{H}^{q}\left(\mathcal{F}^{\bullet}\right)_{x} \cong H^{0}\left(S ; \mathcal{H}^{q}\left(\mathcal{F}^{\bullet}\right)\right)$, for any $x \in S$.

Now let $e^{k, l}$ be the Euler-characteristic of Hodge-type $(k, l)$, i.e., for a bounded complex $K^{\bullet}$ of mixed Hodge structures

$$
e^{k, l}\left(\left[K^{\bullet}\right]\right)=\sum_{i}(-1)^{i} h^{k, l}\left(\left[K^{i}\right]\right) .
$$

Since all differentials in (2.4) are mixed Hodge structure morphisms, an argument similar to that in Lemma 2.6, yields that

$$
\left.e^{k, l}\left(\left[\mathbb{H}^{\bullet}\left(S ; M^{\bullet}\right)\right)\right]\right)=\sum_{r+t=k, s+u=l} e^{r, s}\left(\left[H^{\bullet}(S)\right]\right) \cdot e^{t, u}\left(\left[V^{\bullet}\right]\right) .
$$

Formula (2.18) follows by noting that

$$
\chi_{y}\left(\left[K^{\bullet}\right]\right)=\sum_{k, l} e^{k, l}\left(\left[K^{\bullet}\right]\right) \cdot(-y)^{k} .
$$

Formula (2.19) follows similarly, by working instead with the spectral sequence (2.5). 
Remark 2.19. It follows from the discussion in $\oint 2$, that for any $x \in S$, we have:

$$
\chi_{y}\left(\left[\mathcal{H}^{\bullet}\left(\mathcal{F}^{\bullet}\right)_{x}\right]\right)=\chi_{y}\left(\left[i_{x}^{*} \mathcal{F}^{\bullet}\right]\right),
$$

where $i_{x}:\{x\} \hookrightarrow S$ is the inclusion of the point.

Altogether, Corollary 2.16 and Proposition 2.18 yield the following global-to-local formula:

Theorem 2.20. Let $\mathcal{S}$ be the set of components of strata of an algebraic Whitney stratification of the complex algebraic variety $Z$. Assume that for $M^{\bullet} \in D^{b} M H M(Z)$ with underlying complex $\mathcal{F}^{\bullet} \in D_{c}^{b}(Z)$, the local systems $\mathcal{H}^{j}\left(\mathcal{F}^{\bullet}\right)$ are constant on each pure stratum $S \in \mathcal{S}$ for each $j \in \mathbb{Z}$, e.g. $\pi_{1}(S)=0$ for all $S \in \mathcal{S}$. Then

$$
\chi_{y}\left(\left[\mathbb{H}_{c}^{\bullet}\left(Z ; M^{\bullet}\right)\right]\right)=\sum_{S \in \mathcal{S}} \chi_{y}^{c}(S) \cdot \chi_{y}\left(\left[\mathcal{H}^{\bullet}\left(\mathcal{F}^{\bullet}\right)_{x_{S}}\right]\right),
$$

for some points $x_{S} \in S$.

\section{A Hodge-theoretic analogue of the Riemann-Hurwitz formula}

In this section, we apply the above formalism and properties of $\chi_{y}$-genera to the study of Hodge-type invariants for a projective morphism onto a curve. We first recall the definition of nearby and vanishing cycle functors.

3.1. Vanishing and nearby cycles. Let $X$ be a (separated and reduced) complex analytic space of dimension $n+1$, and $f: X \rightarrow \Delta$ be a holomorphic map onto the unit disc in $\mathbb{C}$, which is smooth over the punctured disc $\Delta^{*}$. The total space $X$ is homotopy equivalent to $X_{0}=f^{-1}(0)$ by a fiber-preserving retraction $r: X \rightarrow X_{0}$. So the inclusion $i_{t}: X_{t}=$ $f^{-1}(t) \hookrightarrow X$ followed by this retraction yields the specialization map $r_{t}: X_{t} \rightarrow X_{0}$.

Let $i_{0}: X_{0} \hookrightarrow X$ be the inclusion map, and define the canonical fiber $X_{\infty}$ by

$$
X_{\infty}:=X \times_{\Delta^{*}} \hbar
$$

where $\hbar$ is the complex upper-half plane (that is, the universal cover of the punctured disc via the map $z \mapsto \exp (2 \pi i z))$. Then $X_{\infty}$ is homotopy equivalent to any smooth fiber $X_{t}$ of $f$. Let $k: X_{\infty} \rightarrow X$ be the induced map.

Definition 3.1. The nearby cycle complex is defined by

$$
\psi_{f}\left(\mathbb{Q}_{X}\right):=i_{0}^{*} R k_{*} k^{*} \mathbb{Q}_{X} \in D_{c}^{b}\left(X_{0}\right) .
$$

By using a resolution of singularities, it can be shown that $\psi_{f}\left(\mathbb{Q}_{X}\right)=R r_{t_{*}} \mathbb{Q}_{X_{t}}$, for $t \neq 0$ small enough. The vanishing cycle complex $\phi_{f}\left(\mathbb{Q}_{X}\right) \in D_{c}^{b}\left(X_{0}\right)$ is the cone on the comparison morphism $\mathbb{Q}_{X_{0}}=i_{0}^{*} \mathbb{Q}_{X} \rightarrow \psi_{f}\left(\mathbb{Q}_{X}\right)$, that is, there exists a canonical morphism can $: \psi_{f}\left(\mathbb{Q}_{X}\right) \rightarrow \phi_{f}\left(\mathbb{Q}_{X}\right)$ such that

$$
i_{0}^{*} \mathbb{Q}_{X} \rightarrow \psi_{f}\left(\mathbb{Q}_{X}\right) \stackrel{\operatorname{can}}{\rightarrow} \phi_{f}\left(\mathbb{Q}_{X}\right) \stackrel{[1]}{\rightarrow}
$$

is a distinguished triangle in $D_{c}^{b}\left(X_{0}\right)$. 
In fact, by replacing $\mathbb{Q}_{X}$ by any complex in $D_{c}^{b}(X)$, we obtain in this way functors $\psi_{f}, \phi_{f}: D_{c}^{b}(X) \rightarrow D_{c}^{b}\left(X_{0}\right)$. It is well-known that if $X$ is locally a complete intersection (e.g., $X$ is smooth), then $\psi_{f} \mathbb{Q}_{X}[n]$ and $\phi_{f} \mathbb{Q}_{X}[n]$ are perverse complexes. This is just a particular case of the fact that the shifted functors ${ }^{p} \psi_{f}:=\psi_{f}[-1]$ and ${ }^{p} \phi_{f}:=\phi_{f}[-1]$ take perverse sheaves on $X$ into perverse sheaves on the central fiber $X_{0}$ (cf. [40], Thm. 6.0.2).

Remark 3.2. The above construction of the vanishing and nearby cycles comes up in the following global context (for details, see [21], §4.2). Let $X$ be a complex algebraic (resp. analytic) variety, and $f: X \rightarrow \mathbb{C}$ a non-constant regular (resp. analytic) function. Then for any $t \in \mathbb{C}$, one can construct functors

$$
\mathcal{F}^{\bullet} \in D_{c}^{b}(X) \mapsto \psi_{f-t}\left(\mathcal{F}^{\bullet}\right), \phi_{f-t}\left(\mathcal{F}^{\bullet}\right) \in D_{c}^{b}\left(X_{t}\right)
$$

where $X_{t}=f^{-1}(t)$ is assumed to be a non-empty hypersurface, by simply repeating the above considerations for the function $f-t$ restricted to a tube $T\left(X_{t}\right):=f^{-1}(\Delta)$ around the fiber $X_{t}$ (here $\Delta$ is a small disc centered at $t$ ). At least in the algebraic context, the tube (which is an analytic space) can be chosen so that $f: T\left(X_{t}\right) \backslash X_{t} \rightarrow \Delta^{*}$ is a topologically locally trivial fibration, where $\Delta^{*}:=\Delta \backslash\{t\}$. In the analytic context, for this to be true one needs to assume, for example, that $f$ is proper on the tube $T\left(X_{t}\right)$.

Of particular importance is the fact that the nearby and vanishing functors can be defined at the level of Saito's mixed Hodge modules [36, 37]. More precisely, if $f$ is a non-constant regular (resp. holomorphic) function on the complex algebraic (resp. analytic) space $X$, then one has functors $\psi_{f}, \phi_{f}: M H M(X) \rightarrow M H M\left(X_{0}\right)$, compatible with the corresponding perverse cohomological functors on the underlying perverse sheaves by the functor

$$
\text { rat }: M H M(X) \rightarrow \operatorname{Perv}_{\mathbb{Q}}(X)
$$

which assigns to a mixed Hodge module the underlying $\mathbb{Q}$-perverse sheaf. In other words, rat $\circ \psi_{f}={ }^{p} \psi_{f} \circ$ rat, and similarly for $\phi_{f}$. As a consequence of this fact, if $X$ is smooth, for each $x \in X_{0}$ we have canonical mixed Hodge structures on the groups

$$
H^{j}\left(M_{x} ; \mathbb{Q}\right)=\mathcal{H}^{j}\left(\psi_{f} \mathbb{Q}_{X}\right)_{x}, \quad \tilde{H}^{j}\left(M_{x} ; \mathbb{Q}\right)=\mathcal{H}^{j}\left(\phi_{f} \mathbb{Q}_{X}\right)_{x},
$$

where $M_{x}$ denotes the Milnor fiber of $f$ at $x$.

By the identification in (3.2), we note that $\operatorname{Supp}\left(\phi_{f} \mathbb{Q}_{X}\right) \subset \operatorname{Sing}\left(X_{0}\right)$ (see [21], Example 4.2.6 and Prop. 4.2.8). Then we have the following vanishing result (e.g., see [40], Example 6.0.13), whose proof we include here for completeness of the exposition:

Lemma 3.3. Assume $X$ is smooth and $f: X \rightarrow \Delta$ is proper. If $s:=\operatorname{dim}_{\mathbb{C}} \operatorname{Sing}\left(X_{0}\right)$, then

$$
\mathbb{H}^{j}\left(X_{0}, \phi_{f} \mathbb{Q}_{X}\right)=0, \text { for } j \notin[n-s, n+s] \text {. }
$$

Proof. Since $X$ is a smooth complex manifold of dimension $n+1$, it follows that $\mathbb{Q}_{X}[n+1]$ is perverse on $X$. Therefore ${ }^{p} \phi_{f} \mathbb{Q}_{X}[n+1]=\phi_{f} \mathbb{Q}_{X}[n] \in \operatorname{Perv}\left(X_{0}\right)$. If $\Sigma:=\operatorname{Sing}\left(X_{0}\right)$, then $\operatorname{Supp}\left(\phi_{f} \mathbb{Q}_{X}\right) \subset \Sigma$ yields that $\left.\phi_{f} \mathbb{Q}_{X}[n]\right|_{\Sigma} \in \operatorname{Perv}(\Sigma)$, see [21], Cor. 5.2.5. The proof follows since (noting that $X_{0}$ and $\Sigma$ are compact)

$$
\mathbb{H}^{j}\left(X_{0}, \phi_{f} \mathbb{Q}_{X}\right) \cong \mathbb{H}^{j-n}\left(X_{0}, \phi_{f} \mathbb{Q}_{X}[n]\right) \cong \mathbb{H}^{j-n}\left(\Sigma,\left.\phi_{f} \mathbb{Q}_{X}[n]\right|_{\Sigma}\right)=0 \text { for } j-n \notin[-s, s],
$$


where the vanishing follows from [21], Proposition 5.2.20.

3.2. A Riemann-Hurwitz formula for $\chi_{y}$-genera. Let $f: X \rightarrow C$ be a surjective projective algebraic morphisms from a smooth $(n+1)$-dimensional complex algebraic variety onto a smooth algebraic curve. Let $\Sigma(f) \subset C$ be the critical locus of $f$. Then $f$ is a submersion over $C^{*}:=C \backslash \Sigma(f)$, hence locally differentiably trivial (by Ehresmann's fibration theorem). For a point $c \in C$ we let $X_{c}$ denote the fiber $f^{-1}(c)$.

We want to relate the $\chi_{y}^{c}$-genera of $X$ and respectively $C$ via the singularities of $f$, and to obtain a stronger version of Prop. 2.11 in our setting. The outcome is a Hodge-theoretic version of a formula of Iversen, or of the Riemann-Hurwitz formula (e.g., see [21], Cor. 6.2.5, Remark 6.2.6, or [29], (III, 32)), see Theorem 3.4 and Example 3.6 below. The proof uses the additivity of the $\chi_{y}^{c}$-genus, together with the study of genera of singular fibers of $f$ by means of vanishing cycles at a critical value.

Theorem 3.4. Let $f: X \rightarrow C$ be a projective algebraic morphism from a smooth $(n+1)$ dimensional complex algebraic variety onto a non-singular algebraic curve $C$. Let $\Sigma(f) \subset C$ be the set of critical values of $f$, and set $C^{*}=C \backslash \Sigma(f)$. If the action of $\pi_{1}\left(C^{*}\right)$ on the cohomology of the generic fibers $X_{t}$ of $f$ is trivial (e.g., $\left.\pi_{1}\left(C^{*}\right)=0\right)$, then

$$
\chi_{y}^{c}(X)=\chi_{y}^{c}(C) \cdot \chi_{y}^{c}\left(X_{t}\right)-\sum_{c \in \Sigma(f)} \chi_{y}\left(\left[\mathbb{H}^{\bullet}\left(X_{c} ; \phi_{f-c} \mathbb{Q}_{X}\right)\right]\right)
$$

Proof. Under our assumptions, the fibers of $f$ are complex projective varieties, and fibers over points in $C^{*}$ are smooth. By additivity, we can write:

$$
\chi_{y}^{c}(X)=\chi_{y}^{c}\left(X^{*}\right)+\sum_{c \in \Sigma(f)} \chi_{y}\left(X_{c}\right)
$$

where $X^{*}:=f^{-1}\left(C^{*}\right)$. Then by Lemma 2.6, we have that $\chi_{y}^{c}\left(X^{*}\right)=\chi_{y}^{c}\left(C^{*}\right) \chi_{y}\left(X_{t}\right)$, where $X_{t}$ is the smooth (generic) fiber of $f$.

Now let $c \in \Sigma(f)$ be a critical value of $f$ and restrict the morphism to a tube $T\left(X_{c}\right):=$ $f^{-1}\left(\Delta_{c}\right)$ around the singular fiber $X_{c}$, where $\Delta_{c}$ denotes a small disc in $C$ centered at $c$. By our assumptions, $f: T\left(X_{c}\right) \rightarrow \Delta_{c}$ is a proper holomorphic function, smooth over $\Delta_{c}^{*}$, with fibers complex projective varieties, that is, a one-parameter degeneration of complex projective varieties. Then there is a long exact sequence of mixed Hodge structures (e.g., see [32], Thm. 1.1):

$$
\cdots \rightarrow H^{j}\left(X_{c} ; \mathbb{Q}\right) \rightarrow \mathbb{H}^{j}\left(X_{c} ; \psi_{f-c} \mathbb{Q}_{X}\right) \rightarrow \mathbb{H}^{j}\left(X_{c} ; \phi_{f-c} \mathbb{Q}_{X}\right) \rightarrow \cdots,
$$

where $\mathbb{H}^{j}\left(X_{c} ; \psi_{f-c} \mathbb{Q}_{X}\right)$ carries the limit mixed Hodge structure defined on the cohomology of the canonical fiber (usually denoted $X_{\infty}$ ) of the one-parameter degeneration $f: T\left(X_{c}\right) \rightarrow$ $\Delta_{c}$ (e.g., see [34], §11.2). However, a consequence of the definition of the limit mixed Hodge structure is that (cf. [34], Cor. 11.25)

$$
\operatorname{dim}_{\mathbb{C}} F^{p} H^{j}\left(X_{\infty} ; \mathbb{C}\right)=\operatorname{dim}_{\mathbb{C}} F^{p} H^{j}\left(X_{t} ; \mathbb{C}\right),
$$


where $X_{t}$ is the generic fiber of the family (and of $f$ ). Therefore,

$$
\chi_{y}\left(X_{\infty}\right):=\chi_{y}\left(\left[\mathbb{H}^{\bullet}\left(X_{c} ; \psi_{f-c} \mathbb{Q}_{X}\right)\right]\right)=\chi_{y}\left(X_{t}\right) .
$$

With this observation, from (3.4) we obtain that for a critical value $c$ of $f$ the following holds:

$$
\begin{aligned}
\chi_{y}\left(X_{c}\right) & =\chi_{y}\left(\left[\mathbb{H}^{\bullet}\left(X_{c} ; \psi_{f-c} \mathbb{Q}_{X}\right)\right]\right)-\chi_{y}\left(\left[\mathbb{H}^{\bullet}\left(X_{c} ; \phi_{f-c} \mathbb{Q}_{X}\right)\right]\right) \\
& =\chi_{y}\left(X_{t}\right)-\chi_{y}\left(\left[\mathbb{H}^{\bullet}\left(X_{c} ; \phi_{f-c} \mathbb{Q}_{X}\right)\right]\right) .
\end{aligned}
$$

By additivity and Lemma 2.6, this yields (3.3).

Remark 3.5. The key point in the proof of the above theorem was to observe that in a one-parameter degeneration of complex projective manifolds the $\chi_{y}$-genus of the canonical fiber coincides with the $\chi_{y}$-genus of the generic fiber of the family. Note that this fact is not true for the corresponding $E$-polynomials, since, while the Hodge structure on the cohomology of the generic fiber is pure, the limit mixed Hodge structure on the cohomology of the canonical fiber carries the monodromy weight filtration.

Example 3.6. If $X$ is smooth and $f$ has only isolated singularities, then

$$
\chi_{y}^{c}(X)=\chi_{y}^{c}(C) \chi_{y}^{c}\left(X_{t}\right)+(-1)^{n+1} \sum_{x \in \operatorname{Sing}(f)} \chi_{y}\left(\left[\tilde{H}^{n}\left(M_{x} ; \mathbb{Q}\right)\right]\right),
$$

where $M_{x}$ is the Milnor fiber of $f$ at $x$. Indeed, if $f$ has only isolated singular points, then each critical fiber $X_{c}$ has only isolated singularities and the corresponding vanishing cycles $\phi_{f-c} \mathbb{Q}_{X}$ are supported only at these points. Then (3.5) follows from the identification in (3.2) and the vanishing of Lemma 3.3.

Remark 3.7. In the special case of the Euler characteristic $\chi=\chi_{-1}$, the formula in Theorem 3.4 and in the example above holds for any proper analytic morphism onto a curve, without any assumption on the monodromy (see [21], Cor. 6.2.5). This follows from the multiplicativity of the Euler characteristic $\chi$ under fibrations, the additivity of compactly supported Euler characteristic $\chi_{c}$, and from the fact that $\chi=\chi_{c}$ (cf. [22], pp. 141-142).

We will extend the formula of Example 3.6 to the case of general singularities. By (3.3), it suffices to restrict $f$ over a small disc $\Delta_{c}$ centered at a critical value $c \in \Sigma(f)$ and to study the polynomial $\chi_{y}\left(\left[\mathbb{H}^{\bullet}\left(X_{c} ; \phi_{f-c} \mathbb{Q}_{X}\right)\right]\right)$. Recall that the fibers of $f$ are complex projective algebraic varieties, which are smooth over points in $\Delta_{c} \backslash\{c\}$.

Fix an algebraic Whitney stratification of $X_{c}$ with respect to which $\phi_{f-c} \mathbb{Q}_{X}$ is constructible. For each $q \in \mathbb{Z}$ and each pure stratum $S \subset \operatorname{Sing}\left(X_{c}\right), \mathcal{H}^{q}\left(\phi_{f-c} \mathbb{Q}_{X}\right)$ is a local coefficient system on $S$ with stalk $\tilde{H}^{q}\left(M_{S} ; \mathbb{Q}\right)$, where $M_{S}$ is the local Milnor fibre at a point in $S$. Then, according to Theorem 2.20 , for $M^{\bullet}=\phi_{f} \mathbb{Q}_{X}[n]$ and by assuming trivial monodromy along all strata $S \subset \operatorname{Sing}\left(X_{c}\right)$, we obtain

$$
\chi_{y}\left(\left[\mathbb{H}^{\bullet}\left(X_{c} ; \phi_{f-c} \mathbb{Q}_{X}\right)\right]\right)=\sum_{S \subset \operatorname{Sing}\left(X_{c}\right)} \chi_{y}^{c}(S) \cdot \chi_{y}\left(\left[\tilde{H}^{\bullet}\left(M_{S} ; \mathbb{Q}\right)\right]\right),
$$


where $M_{S}$ is the local Milnor fibre of $f$ at a point in $S$.

All these facts yield the following general result:

Corollary 3.8. Let $f: X \rightarrow C$ be a projective algebraic morphism from a smooth $(n+1)$ dimensional complex algebraic variety onto a non-singular algebraic curve $C$. Let $\Sigma(f) \subset C$ be the set of critical values of $f$, and set $C^{*}=C \backslash \Sigma(f)$. Assume each special fiber $X_{c}$ has an algebraic stratification with respect to which the corresponding vanishing cycle complex is constructible, and moreover the monodromy along each pure stratum is trivial. If the action of $\pi_{1}\left(C^{*}\right)$ on the cohomology of the generic fibers $X_{t}$ of $f$ is trivial, then

$$
\chi_{y}^{c}(X)=\chi_{y}^{c}(C) \cdot \chi_{y}^{c}\left(X_{t}\right)-\sum_{c \in \Sigma(f)} \sum_{S \subset \operatorname{Sing}\left(X_{c}\right)} \chi_{y}^{c}(S) \cdot \chi_{y}\left(\left[\tilde{H}^{\bullet}\left(M_{S} ; \mathbb{Q}\right)\right]\right)
$$

\section{The presence of monodromy. An Atiyah-Meyer formula for the $\chi_{y}$-GENUS.}

In this section we prove a Hodge-theoretic analogue (in the category of complex algebraic varieties) of the Atiyah-Meyer formula for the signature of fibre bundles in the presence of monodromy [2, 31]. Though some of these results may be true in a greater generality, for simplicity we work in the projective algebraic context.

4.1. Hirzebruch classes of complex projective manifolds and the HirzebruchRiemann-Roch theorem. Recall that if $X$ is a smooth complex projective variety, its Hirzebruch class $\widetilde{T}_{y}^{*}\left(T_{X}\right)$ corresponds to the (un-normalized) power series

$$
\widetilde{Q}_{y}(\alpha):=\frac{\alpha\left(1+y e^{-\alpha}\right)}{1-e^{-\alpha}} \in \mathbb{Q}[y][[\alpha]], \quad \widetilde{Q}_{y}(0)=1+y .
$$

In fact,

$$
\widetilde{T}_{y}^{*}\left(T_{X}\right):=t d^{*}(X) \cup c h^{*}\left(\lambda_{y}\left(T_{X}^{*}\right)\right)
$$

where $t d^{*}(X)$ is the total Todd class of $X, c h^{*}$ is the Chern character, and $\lambda_{y}\left(T_{X}^{*}\right):=$ $\sum_{p} \Lambda^{p} T_{X}^{*} \cdot y^{p}$ is the total $\lambda$-class of (the cotangent bundle of) $X$. Hirzebruch's class appears in the generalized Hirzebruch-Riemann-Roch theorem (cf. [26], §21.3, but for the version needed here see also [45, 8]), which asserts that if $E$ is a holomorphic vector bundle on $X$ then the $\chi_{y}$-characteristic of $E$, which is defined by

$$
\chi_{y}(X, E):=\sum_{p \geq 0} \chi\left(X, E \otimes \Lambda^{p} T_{X}^{*}\right) \cdot y^{p}=\sum_{p \geq 0}\left(\sum_{i \geq 0}(-1)^{i} \operatorname{dim} H^{i}\left(X, \Omega(E) \otimes \Lambda^{p} T_{X}^{*}\right)\right) \cdot y^{p},
$$

with $T_{X}^{*}$ the cotangent bundle of $X$ and $\Omega(E)$ the coherent sheaf of germs of sections of $E 2$, can in fact be expressed in terms of the Chern classes of $E$ and the tangent bundle of $X$,

\footnotetext{
${ }^{2}$ For $X$ smooth and projective, $\chi_{y}\left(X, \mathcal{O}_{X}\right)$ agrees with the Hodge-theoretic $\chi_{y}$-genus defined in the first part of this paper. Indeed, by Deligne's theory, one has the following equality for the Hodge numbers: $h^{p, q}=\operatorname{dim}_{\mathbb{C}} H^{q}\left(X, \Lambda^{p} T_{X}^{*}\right)$.
} 
or more precisely

$$
\chi_{y}(X, E)=\int_{X}\left(c h^{*}(E) \cup \widetilde{T}_{y}^{*}\left(T_{X}\right)\right) \cap[X] .
$$

In particular, if $E=\mathcal{O}_{X}$ we have that

$$
\chi_{y}(X)=\int_{X} \widetilde{T}_{y}^{*}\left(T_{X}\right) \cap[X] .
$$

Also note that the value $y=0$ in (4.1) yields the classical Hirzebruch-Riemann-Roch theorem (in short, HRR) for the holomorphic Euler characteristic of $E$, that is (cf. [26]),

$$
\chi(X, E)=\int_{X}\left(c h^{*}(E) \cup t d^{*}(X)\right) \cap[X] .
$$

4.2. $\chi_{y}$-genera of smooth projective families. Let $f: E \rightarrow B$ be a smooth proper map of smooth projective varieties. By Ehresmann's theorem, $f$ is a differentiable fibration. The cohomology groups $H^{k}\left(E_{b}\right)$ of fibers fit into a local system $R^{k} f_{*} \mathbb{Q}_{E}$. By a result of Griffiths, this local system underlies a weight $k$ (geometric) variation of Hodge structures on $B$ such that the Hodge structure at $b \in B$ is just the Hodge structure we have on $H^{k}\left(E_{b}\right)$. Let

$$
\mathcal{H}_{k}:=R^{k} f_{*} \mathbb{Q}_{E} \otimes_{\mathbb{Q}} \mathcal{O}_{B} .
$$

This is a holomorphic bundle with a flat connection $\nabla: \mathcal{H}_{k} \rightarrow \mathcal{H}_{k} \otimes_{\mathcal{O}_{B}} \Omega_{B}^{1}$, and it admits a finite decreasing filtration $\left\{\mathcal{F}_{k}^{p}\right\}_{p}$ by holomorphic sub-bundles satisfying Griffiths' transversality condition $\nabla\left(\mathcal{F}_{k}^{p}\right) \subset \mathcal{F}_{k}^{p-1} \otimes \Omega_{B}^{1}$. Set $\mathcal{H}^{p, k-p}:=G r_{\mathcal{F}}^{p} \mathcal{H}_{k}$. This is a holomorphic bundle, not necessarily flat, of rank $h_{b}^{p, k-p}:=\operatorname{dim} H^{p, k-p}\left(E_{b} ; \mathbb{C}\right)$. The numbers $h_{b}^{p, k-p}$ for $b \in B$, remain constant in the family since they depend upper-semicontinuously on $b$.

The main result of this section is the following:

Theorem 4.1. Let $f: E \rightarrow B$ be a smooth proper map of smooth complex projective varieties. Then the $\chi_{y}$-genus of $E$ can be computed by the following formula:

$$
\chi_{y}(E)=\int_{B}\left(c h^{*}\left(\chi_{y}(f)\right) \cup \widetilde{T}_{y}^{*}\left(T_{B}\right)\right) \cap[B],
$$

where $\chi_{y}(f):=\sum_{p, q \geq 0}(-1)^{q} \mathcal{H}^{p, q} \cdot y^{p} \in K(B)[y]$ is the $K$-theory $\chi_{y}$-genus of $f$.

Before proving the theorem, we make few remarks and state some immediate consequences.

Remark 4.2. (1) Formula (4.2) shows the deviation from multiplicativity of the $\chi_{y}$-genus of fiber bundles in the presence of monodromy. The right-hand side of (4.2) is a sum of polynomials, one of the summands being $\chi_{y}(B) \cdot \chi_{y}(F)$. Indeed, the zero-dimensional piece of $c h^{*}\left(\chi_{y}(f)\right)$ is $\chi_{y}(F)$. 
(2) Formula (4.2) is the Hodge-theoretic analogue of Atiyah's signature formula (cf. [2], (4.3)) in the complex algebraic setting. Indeed, if $y=1$, then by [45], Remark 3 , and in the notation of [2] $\S 4$,

$$
\widetilde{T}_{1}^{*}\left(T_{B}\right)=\prod_{i=1}^{\operatorname{dim} B} \frac{\alpha_{i}}{\tanh \left(\frac{1}{2} \alpha_{i}\right)}=\widetilde{\mathcal{L}}(B),
$$

where $\alpha_{i}$ are the Chern numbers of the tangent bundle of $B$. Moreover, it is known that $\chi_{1}(E)=\sigma(E)$ is the usual signature (cf. [26]), and in a similar fashion one can show that $(-1)^{q} \mathcal{H}^{p, q}$ is the $K$-theory signature from [2].

An immediate corollary of Theorem 4.1 is the following:

Corollary 4.3. Under the assumptions of the above theorem, if moreover $R^{k} f_{*} \mathbb{Q}_{E}$ is a local system of Hodge structures for each $k$, i.e. the monodromy action of $\pi_{1}(B)$ on the cohomology of the fiber preserves the Hodge filtration, then

$$
\chi_{y}(E)=\chi_{y}(B) \cdot \chi_{y}(F),
$$

where $F$ is the typical fiber of the family.

Proof. Indeed, if $R^{k} f_{*} \mathbb{Q}_{E}$ is a local system of Hodge structures, the Griffiths transversality condition for the flat connection $\nabla: \mathcal{H}_{k} \rightarrow \mathcal{H}_{k} \otimes_{\mathcal{O}_{B}} \Omega_{B}^{1}$ reduces to $\nabla\left(\mathcal{F}_{k}^{p}\right) \subset \mathcal{F}_{k}^{p} \otimes \Omega_{B}^{1}$. It follows that all bundles $\mathcal{F}_{k}^{p}$, whence the bundles $G r_{\mathcal{F}}^{p} \mathcal{H}_{k}=\mathcal{H}^{p, k-p}$, are flat. Since the rational Chern classes in positive degrees of flat bundles are trivial (cf. [28]), we obtain

$$
c h^{*}\left(\sum_{p, q}(-1)^{q} \mathcal{H}^{p, q} y^{p}\right)=\sum_{p, q}(-1)^{q} \operatorname{rank}\left(\mathcal{H}^{p, q}\right) y^{p}=\chi_{y}(F) .
$$

The result follows.

Remark 4.4. The above corollary is false in the non-compact case, e.g., for the Milnor fibration of the cuspidal cupid that was already considered in $\$ 2.4$ (in general, the monodromy of a weighted homogeneous hypersurface singularity is an algebraic morphism, thus induces a morphism of mixed Hodge structures in cohomology).

Remark 4.5. Higher $\chi_{y}$-genera. If $X$ is a smooth projective variety, $\pi:=\pi_{1}(X)$, and $\alpha \in H^{*}(B \pi ; \mathbb{Q})$, we define higher $\chi_{y}$-genera of $X$ by the formula

$$
\chi_{y}^{[\alpha]}(X):=\int_{X}\left(u^{*}(\alpha) \cup \widetilde{T}_{y}^{*}\left(T_{X}\right)\right) \cap[X],
$$

where $u: X \rightarrow B \pi$ is the classifying map of the universal cover of $X$.

Under the assumptions of Corollary [5.7, formula (4.2) can be rephrased in terms of higher $\chi_{y}$-genera as follows. From the classifying space description of each of the bundles $\mathcal{H}^{p, q}$, it is clear that $c h^{*}\left(\mathcal{H}^{p, q}\right)$ is induced from an universal characteristic class $c h^{*}\left(H^{p, q}\right) \in$ $H^{*}(B G L(r ; \mathbb{C}) ; \mathbb{Q})$, where $r=\operatorname{rank} \mathcal{H}^{p, q}$. Moreover, the assumption that the action of $\pi_{1}(B)$ preserves the Hodge filtration, hence the $(p, q)$-type, yields that the classifying map $B \rightarrow B G L(r ; \mathbb{C})$ for $\mathcal{H}^{p, q}$ factors (up to homotopy) as $B \stackrel{u}{\rightarrow} B \pi \stackrel{v}{\rightarrow} B G L(r ; \mathbb{C}$ ), where $u$ 
is the classifying map of the universal cover of $B$, and $v$ is induced by the monodromy action. It follows that $c h^{*}\left(\mathcal{H}^{p, q}\right)=u^{*}\left(v^{*} c h^{*}\left(H^{p, q}\right)\right)$. Set $\alpha_{\pi}^{p, q}:=v^{*} c h^{*}\left(H^{p, q}\right)$. Then the Chern character of the $K$-theory $\chi_{y}$-genus of $f$ can be written as

$$
c h^{*}\left(\chi_{y}(f)\right)=u^{*}\left(\sum_{p, q}(-1)^{q} \alpha_{\pi}^{p, q} \cdot y^{p}\right)
$$

Then in the notation of (4.4) and under the assumptions of Corollary 5.7, formula (4.2) asserts that $\chi_{y}(E)$ can be written as a Hodge polynomial in higher genera of $B$, namely

$$
\chi_{y}(E)=\sum_{p, q}(-1)^{q} \chi_{y}^{\left[\alpha_{T}^{p, q}\right]}(B) \cdot y^{p} .
$$

However, since we assumed that each $\mathcal{H}^{p, q}$ is a flat bundle, we have that $\chi_{y}^{\left[\alpha_{\pi}^{p, q}\right]}(B)=$ $h^{p, q}(F) \cdot \chi_{y}(B)$, and the equation (4.5) yields the multiplicativity of Corollary 5.7.

We now return to the proof of Theorem 4.1,

Proof. Recall from $\$ 2.3$ that the Leray spectral sequence of the map $f$, that is,

$$
E_{2}^{p, q}=H^{p}\left(B, R^{q} f_{*} \mathbb{Q}_{E}\right) \Longrightarrow H^{p+q}(E),
$$

is a spectral sequence of mixed Hodge structures. In fact all mixed Hodge structures involved in the case of a smooth projective family are pure (and by a result of Deligne, the Leray spetral sequence of $f$ degenerates at $E_{2}$ ). Indeed, the local systems $R^{q} f_{*} \mathbb{Q}_{E}$ underlie geometric variations of Hodge structures, thus admissible in the sense of Steenbrink-ZuckerKashiwara (see [34], Theorem 14.49 and the references therein), and it is known (e.g., from Saito's work [37], see also [34], Theorem 14.50) that the cohomology of a smooth projective variety with coefficients in such a variation admits a pure Hodge structure.

By definition, we have

$$
\chi_{y}(E)=\sum_{i, p}(-1)^{i} \operatorname{dim} G r_{F}^{p} H^{i}(E ; \mathbb{C}) \cdot(-y)^{p}=\sum_{p} \chi^{p}(E) \cdot(-y)^{p},
$$

where we let $\chi^{p}(E):=\sum_{i}(-1)^{i} \operatorname{dim} G r_{F}^{p} H^{i}(E ; \mathbb{C})$ be the Euler characteristic associated to the exact functor $G r_{F}^{p}$. Since the differentials in the Leray spectral sequence of $f$ are morphisms of (mixed) Hodge structures, thus strict with respect to the Hodge filtrations, it follows that

$$
\begin{aligned}
\chi^{p}(E) & =\sum_{k, l}(-1)^{k+l} \operatorname{dim} G r_{F}^{p} H^{k}\left(B, R^{l} f_{*} \mathbb{C}_{E}\right) \\
& =\sum_{l}(-1)^{l}\left(\sum_{k}(-1)^{k} \operatorname{dim} G r_{F}^{p} H^{k}\left(B, R^{l} f_{*} \mathbb{C}_{E}\right)\right) \\
& =\sum_{l}(-1)^{l} \chi^{p}\left(B, R^{l} f_{*} \mathbb{Q}_{E}\right) .
\end{aligned}
$$


Therefore,

$$
\begin{aligned}
\chi_{y}(E) & =\sum_{p}\left(\sum_{l}(-1)^{l} \chi^{p}\left(B, R^{l} f_{*} \mathbb{Q}_{E}\right)\right) \cdot(-y)^{p} \\
& =\sum_{l}(-1)^{l}\left(\sum_{p} \chi^{p}\left(B, R^{l} f_{*} \mathbb{Q}_{E}\right) \cdot(-y)^{p}\right) \\
& =\sum_{l}(-1)^{l} \chi_{y}\left(B, R^{l} f_{*} \mathbb{Q}_{E}\right) .
\end{aligned}
$$

So, we reduced the problem to the following setting, which is a Hodge-theoretic analogue of the situation considered by Meyer [31]: $B$ is a smooth projective variety, $\mathbb{V}^{l}:=R^{l} f_{*} \mathbb{Q}_{E}$ is a geometric variation of pure Hodge structures of weight $l$ on $B$ (in fact, for what follows, one may replace $\mathbb{V}^{l}$ by a weight $l$ polarized variation of Hodge structures on $B$ ), and we consider the $\chi_{y}$-genus of $B$ twisted by $\mathbb{V}^{l}$, that is, $\chi_{y}\left(B, \mathbb{V}^{l}\right)$, which encodes the Hodge numbers of the Hodge structures on the cohomology groups $H^{k}\left(B ; \mathbb{V}^{l}\right), k \in \mathbb{Z}_{\geq 0}$.

If we let, as before, $\mathcal{H}_{l}:=\mathbb{V}^{l} \otimes_{\mathbb{Q}} \mathcal{O}_{B}$ be the flat bundle associated to $\mathbb{V}^{l}$, then we have an isomorphism

$$
H^{k}\left(B ; \mathbb{V}^{l} \otimes \mathbb{C}\right) \cong \mathbb{H}^{k}\left(B ; \Omega_{B}^{\bullet} \otimes_{\mathcal{O}_{B}} \mathcal{H}_{l}\right),
$$

and the Hodge filtration on $H^{k}\left(B ; \mathbb{V}^{l} \otimes \mathbb{C}\right)$ is induced by the filtration $F^{\bullet}$ on the de Rham complex that is defined by Griffiths transversality

$$
F^{p}\left(\Omega_{B}^{\bullet} \otimes_{\mathcal{O}_{B}} \mathcal{H}_{l}\right):=\left[\mathcal{F}_{l}^{p} \mathcal{H}_{l} \stackrel{\nabla}{\rightarrow} \Omega_{B}^{1} \otimes \mathcal{F}_{l}^{p-1} \mathcal{H}_{l} \stackrel{\nabla}{\rightarrow} \ldots \stackrel{\nabla}{\rightarrow} \Omega_{B}^{i} \otimes \mathcal{F}_{l}^{p-i} \mathcal{H}_{l} \stackrel{\nabla}{\rightarrow} \ldots\right]
$$

The associated graded is the complex

$$
\operatorname{Gr}_{F}^{p}\left(\Omega_{B}^{\bullet} \otimes_{\mathcal{O}_{B}} \mathcal{H}_{l}\right)=\left(\Omega_{B}^{\bullet} \otimes_{\mathcal{O}_{B}} G r_{\mathcal{F}}^{p-\bullet} \mathcal{H}_{l}, G r_{F} \nabla\right)
$$

with the induced differential.

Then

$$
\begin{aligned}
\chi^{p}\left(B, \mathbb{V}^{l}\right) & =\sum_{k}(-1)^{k} \operatorname{dim} G r_{F}^{p} H^{k}\left(B, \mathbb{V}^{l} \otimes \mathbb{C}\right) \\
& =\sum_{k}(-1)^{k} \operatorname{dim} G r_{F}^{p} \mathbb{H}^{k}\left(B ; \Omega_{B}^{\bullet} \otimes_{\mathcal{O}_{B}} \mathcal{H}_{l}\right) \\
& \stackrel{(*)}{=} \sum_{k}(-1)^{k} \operatorname{dim} \mathbb{H}^{k}\left(B ; G r_{F}^{p}\left(\Omega_{B}^{\bullet} \otimes_{\mathcal{O}_{B}} \mathcal{H}_{l}\right)\right) \\
& =\chi\left(B, \Omega_{B}^{\bullet} \otimes_{\mathcal{O}_{B}} G r_{\mathcal{F}}^{p-\bullet} \mathcal{H}_{l}\right),
\end{aligned}
$$

where $(*)$ follows from the fact proved by M. Saito that $\left(\mathbb{V}^{l}, \Omega_{B}^{\bullet} \otimes_{\mathcal{O}_{B}} \mathcal{H}_{l}\right)$ is a cohomological Hodge complex in the sense of Deligne (see also [34], Theorem 10.9 and Theorem 3.18 (iv)).

The last term in the above equality can be computed by using the invariance of the Euler characteristic under spectral sequences. In general, if $\mathcal{K}^{\bullet}$ is a complex of sheaves on 
a topological space $B$, then there is the following spectral sequence calculating its hypercohomology (e.g., see [21], §2.1):

$$
E_{1}^{i, j}=H^{j}\left(B, \mathcal{K}^{i}\right) \Longrightarrow \mathbb{H}^{i+j}\left(B ; \mathcal{K}^{\bullet}\right) .
$$

Assuming $\chi\left(B, \mathcal{K}^{\bullet}\right)$ is defined, it can be computed by

$$
\chi\left(B, \mathcal{K}^{\bullet}\right)=\sum_{i, j}(-1)^{i+j} \operatorname{dim} H^{j}\left(B, \mathcal{K}^{i}\right)=\sum_{i}(-1)^{i} \chi\left(B ; \mathcal{K}^{i}\right)
$$

Therefore the twisted $\chi_{y}$-genus $\chi_{y}\left(B, \mathbb{V}^{l}\right)$ can be computed as follows (where we neglect the cup product symbol or replace it by "." where there is no danger of confusion):

$$
\begin{aligned}
\chi_{y}\left(B, \mathbb{V}^{l}\right) & =\sum_{p} \chi^{p}\left(B, \mathbb{V}^{l}\right) \cdot(-y)^{p} \\
& =\sum_{p} \chi\left(B, \Omega_{B}^{\bullet} \otimes_{\mathcal{O}_{B}} G r_{\mathcal{F}}^{p-\bullet} \mathcal{H}_{l}\right) \cdot(-y)^{p} \\
& =\sum_{i, p}(-1)^{i} \chi\left(B, \Omega_{B}^{i} \otimes G r_{\mathcal{F}}^{p-i} \mathcal{H}_{l}\right) \cdot(-y)^{p} \\
& \stackrel{(H R R)}{=} \sum_{i, p}(-1)^{i}\left(\int_{B} c h^{*}\left(G r_{\mathcal{F}}^{p-i} \mathcal{H}_{l}\right) c h^{*}\left(\Omega_{B}^{i}\right) t d^{*}(B) \cap[B]\right) \cdot(-y)^{p} \\
& =\int_{B} \sum_{i, p}\left(c h^{*}\left(G r_{\mathcal{F}}^{p-i} \mathcal{H}_{l}\right) \cdot(-y)^{p-i}\right) \cdot\left(c h^{*}\left(\Omega_{B}^{i}\right) t d^{*}(B) \cdot y^{i}\right) \cap[B] \\
& =\int_{B}\left(\sum_{s} c h^{*}\left(G r_{\mathcal{F}}^{s} \mathcal{H}_{l}\right) \cdot(-y)^{s}\right) \cdot\left(t d^{*}(B) \sum_{i} c h^{*}\left(\Omega_{B}^{i}\right) \cdot y^{i}\right) \cap[B] \\
& =\int_{B}\left(\sum_{s} c h^{*}\left(G r_{\mathcal{F}}^{s} \mathcal{H}_{l}\right) \cdot(-y)^{s}\right) \cdot t d^{*}(B) c h^{*}\left(\lambda_{y}\left(T_{B}^{*}\right)\right) \cap[B] \\
= & \int_{B}\left(\sum_{s} c h^{*}\left(G r_{\mathcal{F}}^{s} \mathcal{H}_{l}\right) \cdot(-y)^{s}\right) \cdot \widetilde{T}_{y}^{*}\left(T_{B}\right) \cap[B] .
\end{aligned}
$$

Coming back to the computation of $\chi_{y}(E)$, we obtain that

$$
\begin{aligned}
\chi_{y}(E) & =\sum_{l}(-1)^{l} \chi_{y}\left(B, R^{l} f_{*} \mathbb{Q}_{E}\right) \\
& =\sum_{l}(-1)^{l} \int_{B}\left(\sum_{s} \operatorname{ch}^{*}\left(G r_{\mathcal{F}}^{s} \mathcal{H}_{l}\right) \cdot(-y)^{s}\right) \cdot \widetilde{T}_{y}^{*}\left(T_{B}\right) \cap[B] \\
& =\int_{B}\left(\sum_{l, s}(-1)^{l} \operatorname{ch}^{*}\left(G r_{\mathcal{F}}^{s} \mathcal{H}_{l}\right) \cdot(-y)^{s}\right) \cdot \widetilde{T}_{y}^{*}\left(T_{B}\right) \cap[B]
\end{aligned}
$$




$$
\begin{aligned}
& =\int_{B}\left(\sum_{l, s}(-1)^{l} \operatorname{ch}^{*}\left(\mathcal{H}^{s, l-s}\right) \cdot(-y)^{s}\right) \cdot \widetilde{T}_{y}^{*}\left(T_{B}\right) \cap[B] \\
& =\int_{B}\left(\sum_{p, q}(-1)^{q} \operatorname{ch}^{*}\left(\mathcal{H}^{p, q}\right) \cdot y^{p}\right) \cdot \widetilde{T}_{y}^{*}\left(T_{B}\right) \cap[B] .
\end{aligned}
$$

As an important corollary of the proof of Theorem 4.1 we obtain the following Hodgetheoretic analogue of Meyer's signature formula [31]:

Corollary 4.6. Let $Z$ be a smooth projective variety and $\mathbb{V}$ a polarized variation of Hodge structures on $Z$, with associated flat bundle with "Hodge" filtration $(\mathcal{V}, \mathcal{F} \bullet)$. Then the twisted $\chi_{y}$-genus $\chi_{y}(Z, \mathbb{V})$ can be computed by the formula

$$
\chi_{y}(Z, \mathbb{V})=\int_{Z}\left(\operatorname{ch}^{*}\left(H c_{y}(\mathcal{V})\right) \cup \widetilde{T}_{y}^{*}\left(T_{Z}\right)\right) \cap[Z],
$$

where $H c_{y}(\mathcal{V})$ is the $K$-theory Hodge polynomial characteristic of $\mathcal{V}$ defined by

$$
H c_{y}(\mathcal{V})=\sum_{p} G r_{\mathcal{F}}^{p} \mathcal{V} \cdot(-y)^{p}
$$

Remark 4.7. As stated in [31], Meyer's formula for the signature $\sigma(Z ; \mathcal{L})$ of a Poincaré local system $\mathcal{L}$ (that is, a local system equipped with a nondegenerate bilinear pairing $\mathcal{L} \otimes \mathcal{L} \rightarrow \mathbb{R}_{Z}$ ) on a closed oriented smooth manifold $Z$ of even dimension involves a twisted Chern character and the total $L$-polynomial of $Z$ (as opposed to Atiyah's formula [2], where an un-normalized version of the $L$-polynomial is used). More precisely ([31]),

$$
\sigma(Z ; \mathcal{L})=\int_{Z}\left(\widetilde{c h^{*}}\left([\mathcal{L}]_{K}\right) \cup L(Z)\right) \cap[Z]
$$

where $[\mathcal{L}]_{K}$ is the $K$-theory signature of $\mathcal{L}, L(Z)$ is the total Hirzebruch $L$-polynomial of $Z$, and $\widetilde{c h^{*}}:=c h^{*} \circ \psi^{2}$ is a modified Chern character obtained by composition with the second Adams operation. Similarly, following [27], p.61-62 (see also [41], §6), we can reformulate our Hodge-theoretic Atiyah-Meyer formulae in terms of the normalized Hirzebruch classes $T_{y}^{*}\left(T_{Z}\right)$ corresponding to the power series

$$
Q_{y}(\alpha):=\widetilde{Q}_{y}(\alpha(1+y)) \cdot(1+y)^{-1}=\frac{\alpha(1+y)}{1-e^{-\alpha(1+y)}}-\alpha y \in \mathbb{Q}[y][[\alpha]],
$$

by using instead a modified Chern character, $c h_{(1+y)}^{*}$, whose value on a complex vector bundle $E$ is

$$
c h_{(1+y)}^{*}(E)=\sum_{j=1}^{r k E} e^{\beta_{j}(1+y)}
$$


for $\beta_{j}$ the Chern roots of $E$. (In this notation, Meyer's modified Chern character is simply $c h_{(2)}^{*}$.) For example, in the notations of Corollary 4.6, formula (4.7) is equivalent to

$$
\chi_{y}(Z, \mathbb{V})=\int_{Z}\left(c h_{(1+y)}^{*}\left(H c_{y}(\mathcal{V})\right) \cup T_{y}^{*}\left(T_{Z}\right)\right) \cap[Z] .
$$

A similar formula can be obtained for $\chi_{y}(U ; \mathbb{V})$, the twisted $\chi_{y}$ polynomial associated to the canonical mixed Hodge structure on $H^{*}(U ; \mathbb{V})$, for $U$ any smooth (not necessarily compact) complex variety and $\mathbb{V}$ an admissible variation of mixed Hodge structure on $U$. (The existence of such mixed Hodge structures follows for example from Saito's theory, see also [34], Thm 14.50 and the references therein.) In this case, we need to use the twisted logarithmic de Rham complex associated to the Deligne extension on a good compactification of $U$. More precisely, let $(\mathcal{V}, \nabla)$ be the corresponding vector bundle on $U$ with its flat connection and Hodge filtration. Then we can choose a smooth compactification $j: U \rightarrow Z$ such that $D=Z \backslash U$ is a divisor with normal crossings, and for each half-open interval of length one there is a unique extension of $(\mathcal{V}, \nabla)$ to a vector bundle $\left(\overline{\mathcal{V}}^{I}, \bar{\nabla}^{I}\right)$ with a logarithmic connection on $Z$ such that the eigenvalues of the residues lie in $I$ (cf. $[19]$. If we set $\overline{\mathcal{V}}:=\overline{\mathcal{V}}^{[0,1)}$, then the twisted logarithmic de Rham complex $\Omega_{Z}^{\bullet}(\log D) \otimes \overline{\mathcal{V}}$ is quasi-isomorphic (on $Z$ ) to $R j_{*} \mathbb{V} \otimes \mathbb{C}$, and the filtration $\mathcal{F}^{\bullet}$ on $\mathcal{V}$ extends to a filtration $\overline{\mathcal{F}}^{\bullet} \subset \overline{\mathcal{V}}$ since the variation of mixed Hodge structures was assumed to be admissible. As before, by Griffiths' transversality, we can filter the logarithmic twisted de Rham complex, and Saito proved that this becomes part of a cohomological mixed Hodge complex that calculates $H^{*}(U ; \mathbb{V})$. By repeating the arguments in the proof of Theorem 4.1, we obtain the following formula, analogous to (4.7), involving contributions at infinity (i.e., involving forms on $Z$, with logarithmic poles along $D)$ :

$$
\chi_{y}(U ; \mathbb{V})=\int_{Z}\left(c h^{*}\left(H c_{y}(\overline{\mathcal{V}})\right) \cup c h^{*}\left(\sum_{i} \Omega_{Z}^{i}(\log D) \cdot y^{i}\right) \cup t d^{*}(Z)\right) \cap[Z] .
$$

This explains why under the assumptions of Corollary 5.7, the multiplicativity of the $\chi_{y^{-}}$ genus fails in the non-compact case (cf. Remark 4.4).

4.3. Higher $\chi_{y}$-genera and period domains. In this section, we define higher $\chi_{y}$-genera of variations of Hodge structures which correspond to cohomology classes of the quotients of Griffths period domains (cf. [G]). These higher genera are analogous to the previously considered higher genera corresponding to the cohomology classes of the fundamental group (cf. Remark 4.5), and for some types of variations of Hodge structures coincide with the latter. These classes allow to obtain a formula for the $\chi_{y}$-genus of a fibration in terms of characteristic classes of the base, which yields the multiplicativity in a variety of cases including the case of trivial monodromy group.

Let $(B, \mathbb{V})$ be a pair where $B$ is a Kähler manifold and $\mathbb{V}$ is a (integer) polarized variation of pure Hodge structures of weight $k$. If $V$ is the stalk of $\mathbb{V}$ at a point in $B$, let $\epsilon= \pm 1$ be the type of the bilinear form $Q$ on $V$ (i.e. $Q(x, y)=\epsilon Q(y, x))$, and $\eta_{V}$ be the partition $\operatorname{dim} V=\sum_{p+q=k} h^{p, q}$ where $h^{p, q}=\operatorname{dim} H^{p, q}$. Let $D_{\eta_{V}}$ be the classifying space of pure Hodge 
structures of type $\left(\epsilon, \eta_{V}\right)$. This space is a subset in the flag manifold (consisting of flags in $V$ satisfying the Riemann bilinear relations) and in particular it is the base of the universal flag bundle $\mathcal{F}_{\eta_{V}}:=\cdots \subset \mathcal{F}^{p} \subset \cdots\left(\operatorname{rank} \mathcal{F}^{p}=\sum_{i \leq p} \operatorname{dim} H^{i, k-i}\right)$ having the flags as its fiber, and also for the bundles $\mathcal{H}^{p, k-p}$ which are the quotients $\mathcal{F}^{p} / \mathcal{F}^{p+1}$.

Let $\bar{\Gamma}$ be the monodromy group corresponding to $\mathbb{V}$. This is a subgroup in the subgroup of $G L(\operatorname{dim} V, \mathbf{Z})$ consisting of transformations preserving $Q$. The group $\bar{\Gamma}$ acts on $D_{\epsilon, \eta_{V}}$ and some subgroup $\Gamma \subseteq \bar{\Gamma}$ of finite index acts freely on $D_{\epsilon, \eta_{V}}$. The pair $(B, \mathbb{V})$ defines the period map:

$$
\pi: B \rightarrow D_{\epsilon, \eta_{V}} / \Gamma \text {. }
$$

The action of the group $\Gamma$ on $V$ and $D_{\epsilon, \eta_{V}}$ induces the action on the total space of $\mathcal{F}_{\eta_{V}}$ so that the projection $\mathcal{F}_{\eta_{V}} \rightarrow D_{\epsilon, \eta_{V}}$ is $\Gamma$-equivariant. The latter map induces the locally trivial fibration: $\mathcal{F}_{\eta_{V}} / \Gamma \rightarrow D_{\epsilon, \eta_{V}} / \Gamma$ and, moreover, for any $h^{p, q} \in \eta_{V}$ the bundle $\mathcal{H}^{p, q}$ over $D_{\epsilon, \eta_{V}}$ descends to a bundle over the quotient $D_{\epsilon, \eta_{V}} / \Gamma$.

Definition 4.8. Let $\alpha \in H^{*}\left(D_{\epsilon, \eta_{V}} / \Gamma\right)$. The higher genus $\chi_{y}^{[\alpha]}$ is given by:

$$
\chi_{y}^{[\alpha]}=\pi^{*}(\alpha) \cup \widetilde{T}_{y}(B)[B] .
$$

Among variations of Hodge structures one can single out those for which, in the case $\epsilon=-1$ there are at most two non-vanishing Hodge numbers, and for $\epsilon=+1$ if $p \neq q$ then all $h^{p, q}=0$ except for at most two of them for which one has $h^{p, q}=1$. In this case, the period domain is simply-connected since it is the Siegel upper-half plane for $\epsilon=-1$ and $S O\left(2, h^{p, p}\right) / U(1) \times S O\left(h^{p, p}\right)$, i.e. the quotient by the maximal compact subgroup, for $\epsilon=+1$ (cf. [13], p.145). In this case the period map factors as $B \rightarrow B \pi_{1}(B) \rightarrow D_{\epsilon, \eta_{V}} / \Gamma=B(\Gamma)$ (the latter is the classifying space of $\Gamma$ ), and the $\chi_{y}^{[\alpha]}$ coincides with the higher- $\chi_{y}$ genus considered in [7] (see also Remark [4.5). We shall refer to such variations as topological variations of Hodge structures.

Next we shall consider a geometric variation of Hodge structures arising from a smooth proper map of smooth projective varieties $f: E \rightarrow B$. The Kähler class $\omega_{E}$ of $E$ induces the Kähler class $\omega_{F}$ on each fiber $F$, which is left invariant by the monodromy of this fibration. In particular the Hodge form on $H^{k}(F)$ (given by $Q(\alpha, \beta)=\left(\omega_{F}^{\operatorname{dim} F-2 k} \cup \alpha \cup \beta\right)[F]$ ) is a monodromy invariant. The primitive cohomology of $F$ yields a polarized variation of pure Hodge structures, and the fundamental group $\pi_{1}(B)$ acts via the monodromy representation on $\oplus_{k} H_{\text {prim }}^{k}(F)$ and also on $\prod_{k} D_{(-1)^{k}, \eta_{k}}$, where $\eta_{k}$ is the partition of $\operatorname{dim} H^{k}(F)_{\text {prim }}=$ $\sum_{p+q=k} \operatorname{dim} H^{p, q}(F) \cap H^{k}(F)_{\text {prim }}$. Let $\bar{\Gamma}$ be the quotient on $\pi_{1}(B)$ by the kernel of this action, and $\Gamma$ be a subgroup of finite index acting freely.

Theorem 4.9. Let $f: E \rightarrow B$ be a smooth proper map of smooth projective varieties, and

$$
\pi: B \rightarrow\left(\prod_{k} D_{(-1)^{k}, \eta_{k}}\right) / \Gamma
$$

be the total period map. Let $\pi_{k}$ be the projection of the target of $\pi$ on the $k$-th component, and let $\alpha_{\Gamma}^{p, q}=\pi_{k}^{*}\left(\operatorname{ch}^{*}\left(\mathcal{H}^{p, q}\right)\right)$ be the pull back to the quotient of the total period map of the 
Chern character of the bundle $\mathcal{H}^{p, q}$. Then $\chi_{y}(E)$ is given by the formula (compare with (4.5))

$$
\chi_{y}(E)=\sum_{p, q}(-1)^{q} \chi_{y}^{\left[\alpha_{\Gamma}^{p, q}\right]}(B) \cdot y^{p} .
$$

The proof follows from the formula (4.2) similarly to the way (4.5) was derived from (4.2).

Remark 4.10. If $\Gamma=1$ (i.e., the monodromy group is trivial or finite) we obtain multiplicativity. More generally, if the $H^{p, q}$ are monodromy invariant then the period map is homotopic to the map to a point and again one has multiplicativity.

Remark 4.11. Fibrations for which the fibers are curves or K3 surfaces induce topological variations of Hodge structures and hence the $\chi_{y}$-genus of the total space can be expressed in terms of Novikov's type $\chi_{y}$-genus as in Remark 4.5. On the other hand, for fibrations with fibers of higher dimensions one needs the generalization of the higher $\chi_{y}$-genus except for very special cases.

Remark 4.12. The generalization of the higher $\chi_{y}$-genus given in definition (4.8) has the strong birational invariance property of higher genera corresponding to twisting by the cohomology classes of the fundamental group. More precisely, we have the K-equivalence relation among pairs $\left(B_{1}, \mathbb{V}_{1}\right)$ and $\left(B_{2}, \mathbb{V}_{2}\right)$ generated by the elementary K-equivalence $f_{1}: X \rightarrow B_{1}, f_{2}: X \rightarrow B_{2}$ such that $f_{1}^{*}\left(\mathbb{V}_{1}\right)=f_{2}^{*}\left(\mathbb{V}_{2}\right)$. The monodromy groups $\Gamma$ and total period domains $D$ of $\mathbb{V}_{1}$ and $\mathbb{V}_{2}$ are the same, and for any $\alpha$ in $H^{*}(D / \Gamma)$ the $\chi_{y}^{[\alpha]}$-genera of $\left(B_{i}, \mathbb{V}_{i}\right)$ coincide. This follows from the push-forward formulas in the same way as in [7]. We shall discuss characterization of such generalized twisted $\chi_{y}$ invariants elsewhere.

\section{Atiyah-Meyer type Characteristic Class FORMulae.}

In this section, we present characteristic class versions of the above Atiyah-Meyer formulae for the $\chi_{y}$-genus. The proofs of these characteristic class formulae are much more involved, and make use of Saito's theory of mixed Hodge modules and the construction of the motivic Hirzebruch classes (cf. [8]), which we recall here. For full details on this construction, the reader is advised to consult [8, 10].

Let $Z$ be a complex algebraic variety. Then for any $p \in \mathbb{Z}$ one has a functor of triangulated categories

$$
g r_{p}^{F} D R: D^{b} M H M(Z) \rightarrow D_{c o h}^{b}(Z)
$$

commuting with proper push-down, where $D_{c o h}^{b}(Z)$ is the bounded derived category of sheaves of $\mathcal{O}_{Z}$-modules with coherent cohomology sheaves. If $\mathbb{Q}_{Z}^{H} \in D^{b} M H M(Z)$ denotes the constant Hodge module on $Z$, and if $Z$ is smooth and pure dimensional then $g r_{-p}^{F} D R\left(\mathbb{Q}_{Z}^{H}\right) \simeq \Omega_{Z}^{p}[-p] \in D_{c o h}^{b}(Z)$. The transformations $g r_{p}^{F} D R(M)$ are functors of triangulated categories, so they induce functors on the level of Grothendieck groups. Thus, if $G_{0}(Z) \simeq K_{0}\left(D_{c o h}^{b}(Z)\right)$ denotes the Grothendieck group of coherent sheaves on $Z$, we obtain the following group homomorphism commuting with proper push-down:

$$
g r_{-*}^{F} D R: K_{0}(M H M(Z)) \rightarrow G_{0}(Z) \otimes \mathbb{Z}\left[y, y^{-1}\right],
$$




$$
[M] \mapsto \sum_{p}\left(\sum_{i}(-1)^{i}\left[\mathcal{H}^{i}\left(g r_{-p}^{F} D R(M)\right)\right]\right) \cdot(-y)^{p}
$$

We can now make the following definitions (see [8, 10])

Definition 5.1. The transformation $\widetilde{M H T}$ is defined as the composition of transformations:

$$
\widetilde{M H T}_{y}:=t d_{*} \circ g r_{-*}^{F} D R: K_{0}(M H M(Z)) \rightarrow H_{2 *}^{B M}(Z) \otimes \mathbb{Q}\left[y, y^{-1}\right],
$$

where $t d_{*}$ is the Baum-Fulton-MacPherson Todd class transformation [4, which is linearly extended over $\mathbb{Z}\left[y, y^{-1}\right]$. Note that $\widetilde{M H T}_{y}$ commutes with proper push-forward.

Remark 5.2. Let $K^{0}(Z)$ be the Grothendieck group of complex algebraic vector bundles on $Z$. If $Z$ a smooth complex manifold, the canonical map $K^{0}(Z) \rightarrow G_{0}(Z)$ induced by taking the sheaf of sections is an isomorphism, and the Todd class transformation is explicitly described by $t d_{*}(\cdot)=c h^{*}(\cdot) \cup t d^{*}\left(T_{Z}\right) \cap[Z]$.

Definition 5.3. The Hirzebruch class of an $n$-dimensional complex algebraic variety $Z$ is defined by the formula

$$
\widetilde{T}_{y_{*}}(Z):=\widetilde{M H T}_{y}\left(\left[\mathbb{Q}_{Z}^{H}\right]\right) .
$$

Similarly, if $Z$ is an $n$-dimensional complex algebraic manifold, and $\mathbb{V}$ a polarized variation of Hodge structures on $Z$, we define twisted Hirzebruch characteristic classes

$$
\widetilde{T}_{y_{*}}(Z ; \mathbb{V})=\widehat{M H T}_{y}\left(\left[\mathbb{V}^{H}\right]\right),
$$

where $\mathbb{V}^{H}[n]=\left((\mathcal{V}, \nabla), \mathcal{F}_{-}, \mathbb{V}[n]\right)$ is the smooth mixed Hodge module on $Z$ corresponding to $\mathbb{V}$, with $\mathcal{F}_{-\bullet}:=\mathcal{F}^{\bullet}$ (e.g., see [34], Thm. 14.30 and the references therein).

By [[8], Lemma 3.1 and Theorem 3.1], the following normalization holds: if $Z$ is smooth and pure dimensional, then $\widetilde{T}_{y_{*}}(Z)=\widetilde{T}_{y}^{*}\left(T_{Z}\right) \cap[Z]$, thus $\widetilde{T}_{y_{*}}(Z)$ is an extension to the singular setting of (the Poincaré dual of) the un-normalized Hirzebruch class.

We can now prove the following Meyer-type formula for the twisted Hirzebruch characteristic classes

Theorem 5.4. Let $Z$ be a smooth projective variety of pure dimension $n$, and $\mathbb{V}$ a polarized variation of Hodge structures on $Z$ with associated flat bundle with "Hodge" filtration $\left(\mathcal{V}, \mathcal{F}^{\bullet}\right)$. Then

$$
\widetilde{T}_{y_{*}}(Z ; \mathbb{V})=\left(\operatorname{ch}^{*}\left(H c_{y}(\mathcal{V})\right) \cup \widetilde{T}_{y}^{*}\left(T_{Z}\right)\right) \cap[Z]=\operatorname{ch}^{*}\left(H c_{y}(\mathcal{V})\right) \cap \widetilde{T}_{y_{*}}(Z),
$$

where $H c_{y}(\mathcal{V})$ is the $K$-theory Hodge polynomial characteristic of $\mathcal{V}$.

Proof. Let $\mathbb{V}^{H}[n]=\left((\mathcal{V}, \nabla), \mathcal{F}_{-}, \mathbb{V}[n]\right)$ be the smooth mixed Hodge module on $Z$ corresponding to $\mathbb{V}$, with $\mathcal{F}_{-} \bullet:=\mathcal{F}^{\bullet}$ the increasing filtration on the $\mathcal{D}$-module $\mathcal{V}$. It follows from Saito's work that there is a filtered quasi-isomorphism between $\left(D R\left(\mathbb{V}^{H}\right), F_{-\bullet}\right)$ and 
the usual filtered de Rham complex $\left(\Omega_{Z}^{\bullet}(\mathcal{V}), F^{\bullet}\right)$ with the filtration induced by Griffiths' transversality, that is:

$$
F^{p} \Omega_{Z}^{\bullet}(\mathcal{V}):\left[\mathcal{F}^{p} \stackrel{\nabla}{\rightarrow} \Omega_{Z}^{1} \otimes \mathcal{F}^{p-1} \stackrel{\nabla}{\rightarrow} \cdots \stackrel{\nabla}{\rightarrow} \Omega_{Z}^{i} \otimes \mathcal{F}^{p-i} \stackrel{\nabla}{\rightarrow} \cdots\right] .
$$

Therefore,

$$
\begin{aligned}
\widetilde{T}_{y_{*}}(Z ; \mathbb{V}) & =t d_{*}\left(\sum_{p}\left(\sum_{i}(-1)^{i}\left[\mathcal{H}^{i}\left(g r_{-p}^{F} D R\left(\mathbb{V}^{H}\right)\right)\right]\right) \cdot(-y)^{p}\right) \\
& =t d_{*}\left(\sum_{p}\left(\sum_{i}(-1)^{i}\left[\mathcal{H}^{i}\left(g r_{F}^{p} \Omega_{Z}^{\bullet}(\mathcal{V})\right)\right]\right) \cdot(-y)^{p}\right) \\
& =t d_{*}\left(\sum_{p}\left(\sum_{i}(-1)^{i}\left[\Omega_{Z}^{i} \otimes G r_{\mathcal{F}}^{p-i} \mathcal{V}\right]\right) \cdot(-y)^{p}\right) \\
& =\sum_{p} \sum_{i}(-1)^{i} t d_{*}\left(\left[\Omega_{Z}^{i} \otimes G r_{\mathcal{F}}^{p-i} \mathcal{V}\right]\right) \cdot(-y)^{p} \\
& =\sum_{p} \sum_{i}(-1)^{i} c h^{*}\left(\Omega_{Z}^{i} \otimes G r_{\mathcal{F}}^{p-i} \mathcal{V}\right) \cup t d^{*}(Z) \cap[Z] \cdot(-y)^{p} \\
& =\sum_{p} \sum_{i}\left(c h^{*}\left(G r_{\mathcal{F}}^{p-i} \mathcal{V}\right) \cdot(-y)^{p-i}\right) \cup\left(c h^{*}\left(\Omega_{Z}^{i}\right) \cdot y^{i}\right) \cup t d^{*}(Z) \cap[Z] \\
& =\left(\sum_{q} c h^{*}\left(G r_{\mathcal{F}}^{q} \mathcal{V}\right) \cdot(-y)^{q}\right) \cup\left(\sum_{i} c h^{*}\left(\Omega_{Z}^{i}\right) \cdot y^{i}\right) \cup t d^{*}(Z) \cap[Z] \\
& =c h^{*}\left(H c_{y}(\mathcal{V})\right) \cup c h^{*}\left(\lambda_{y}\left(T_{Z}^{*}\right)\right) \cup t d^{*}(Z) \cap[Z] \\
& =h^{*}\left(H c_{y}(\mathcal{V})\right) \cup \tilde{T}_{y}^{*}\left(T_{Z}\right) \cap[Z],
\end{aligned}
$$

where $(G R R)$ means an application of the classical Grothendieck-Riemann-Roch theorem.

Remark 5.5. More generally, Saito [37] showed that an admissible variation of mixed Hodge structures on a smooth variety $Z$ (e.g., a geometric variation), with underlying local system $\mathcal{L}$, gives rise to a smooth mixed Hodge module on $Z$, with $\mathcal{L}[\operatorname{dim} Z]$ as its underlying perverse sheaf. Then one can define twisted Hirzebruch characteristic classes associated to such admissible variations. Note that Theorem 5.4 remains true in this greater generality, i.e., we can let $\mathbb{V}$ be a geometric variation of Hodge structures, or more generally, an admissible variation of mixed Hodge structures.

Jörg Schürmann [42] communicated to us that the following Atiyah-type result can be obtained as a direct application of the Verdier-Riemann-Roch formula for a smooth proper morphism (cf. [8], Cor. 3.1(3)), if one makes the identification $\mathcal{H}^{p, q} \simeq R^{q} f_{*}\left(\Lambda^{p} T_{f}^{*}\right.$ ), with $T_{f}^{*}$ the dual of the tangent bundle $T_{f}$ to the fibers of $f$ (cf. [34], Proposition 10,28). However, 
the proof we give here is based only on the definition of the Hirzebruch classes and on Theorem 5.4 in the context of geometric variations of Hodge structures.

Theorem 5.6. Let $f: E \rightarrow B$ be a smooth proper map of smooth complex projective varieties. Then the following holds:

$$
f_{*} \widetilde{T}_{y_{*}}(E)=c h^{*}\left(\chi_{y}(f)\right) \cap \widetilde{T}_{y_{*}}(B),
$$

where $\chi_{y}(f)=\sum_{p, q \geq 0}(-1)^{q} \mathcal{H}^{p, q} \cdot y^{p}$ is the $K$-theory $\chi_{y}$-genus of $f$.

Proof. Since $f$ is proper and the transformation $\widetilde{M H T}_{y}$ commutes with proper pushdowns, we first obtain the following:

$$
f_{*} \widetilde{T}_{y_{*}}(E)=f_{*}\left(\widetilde{M H T}_{y}\left(\left[\mathbb{Q}_{E}^{H}\right]\right)\right)=\widetilde{M H T_{y}}\left(\left[f_{*} \mathbb{Q}_{E}^{H}\right]\right) .
$$

Now let $\tau_{\leq}$be the natural truncation on $D^{b} M H M(B)$ with associated cohomology $H^{\bullet}$. Then for any complex $M^{\bullet} \in D^{b} M H M(B)$ we have the identification (e.g., see [21], p. 95-96; [40], Lemma 3.3.1)

$$
\left[M^{\bullet}\right]=\sum_{i \in \mathbb{Z}}(-1)^{i}\left[H^{i}\left(M^{\bullet}\right)\right] \in K_{0}\left(D^{b} M H M(B)\right) \cong K_{0}(M H M(B)) .
$$

In particular, if for any $k \in \mathbb{Z}$ we regard $H^{i+k}\left(M^{\bullet}\right)[-k]$ as a complex concentrated in degree $k$, then

$$
\left[H^{i+k}\left(M^{\bullet}\right)[-k]\right]=(-1)^{k}\left[H^{i+k}\left(M^{\bullet}\right)\right] \in K_{0}(M H M(B)) .
$$

Therefore, if we let $M^{\bullet}=f_{*} \mathbb{Q}_{E}^{H}$, we obtain that

$$
f_{*} \widetilde{T}_{y_{*}}(E)=\sum_{i \in \mathbb{Z}}(-1)^{i} \widetilde{M H T}_{y}\left(\left[H^{i}\left(f_{*} \mathbb{Q}_{E}^{H}\right)\right]\right)=\sum_{i \in \mathbb{Z}}(-1)^{i} \widehat{M H T}_{y}\left(\left[H^{i+\operatorname{dim} B}\left(f_{*} \mathbb{Q}_{E}^{H}\right)[-\operatorname{dim} B]\right]\right) .
$$

Note that $H^{i}\left(f_{*} \mathbb{Q}_{E}^{H}\right) \in M H M(B)$ is the smooth mixed Hodge module on $B$ whose underlying rational complex is (recall that $B$ is smooth)

$$
\operatorname{rat}\left(H^{i}\left(f_{*} \mathbb{Q}_{E}^{H}\right)\right)={ }^{p} \mathcal{H}^{i}\left(R f_{*} \mathbb{Q}_{E}\right)=\left(R^{i-\operatorname{dim} B} f_{*} \mathbb{Q}_{E}\right)[\operatorname{dim} B]
$$

where for the second equality we refer to [34], Example 13.20. In this case, each of the local systems $\mathcal{L}_{s}:=R^{s} f_{*} \mathbb{Q}_{E}$ underlies a geometric variation of Hodge structures.

Altogether, (5.10) becomes

$$
f_{*} \widetilde{T}_{y_{*}}(E)=\sum_{i \in \mathbb{Z}}(-1)^{i} \widetilde{T}_{y_{*}}\left(B ; \mathcal{L}_{i}^{H}\right),
$$

where, by analogy with Definition $5.3, \mathcal{L}_{i}^{H}[\operatorname{dim} B]:=H^{i+\operatorname{dim} B}\left(f_{*} \mathbb{Q}_{E}^{H}\right)$ is the smooth mixed Hodge module whose underlying perverse sheaf is $\mathcal{L}_{i}[\operatorname{dim} B]$.

Our formula (5.6) follows now from Theorem 5.4 and Remark 5.5.

An immediate corollary of Theorem 5.6] is the following extension of [[10], Cor. 3.12], whose proof imitates that of Corollary 5.7. 
Corollary 5.7. Under the assumptions of the above theorem, if moreover $R^{k} f_{*} \mathbb{Q}_{E}$ is a local system of Hodge structures for each $k$, i.e. the monodromy action of $\pi_{1}(B)$ on the cohomology of the fiber preserves the Hodge filtration, then

$$
f_{*} \widetilde{T}_{y_{*}}(E)=\chi_{y}(F) \widetilde{T}_{y_{*}}(B),
$$

where $F$ is the fiber of $f$.

Remark 5.8. It is easy to see that all results for genera in Section 4.2 can be obtained by taking the degree zero parts of formulae in the present section. However, we chose to present the results for genera first, since they can be proven by using only standard facts of classical Hodge theory.

\section{Twisted $\chi_{y}$-Genera And Characteristic ClASSES IN The Singular SEtTing.}

In this section, we discuss possible generalizations of formulae (4.2), (4.7), (5.5) and (5.6) to the singular setting. This is motivated by the considerations in [10], some of which are extended here, and by an extension of the Atiyah-Meyer signature and $L$-class formulae to the singular case, which is due to Banagl, Cappell and Shaneson (cf. [3]).

One possible extension of our Hodge theoretic Atiyah-Meyer formulae to the singular setting makes use of intersection homology. Geometric mapping situations involving singular spaces lead naturally to the study of twisted intersection homology genera and corresponding characteristic classes, e.g., see [11] for a study of twisted signatures and twisted $L$-classes. In $\$ 6.1$, we show how twisted Hodge-theoretic intersection homology genera arise in mapping situations, and we extend some results of [10] concerning the stratified multiplicative property for the $I \chi_{y}$-genus. In $\$ 6.2$, we conjecture an extension of the Meyer-type formulae (4.7) and (5.5) to the singular setting, supply a proof in a special case, and indicate an outline of its proof in general.

6.1. Hodge intersection homology genera and the stratified multiplicative property. Let $f: X \rightarrow Y$ be a proper morphism of complex algebraic variety, and $\mathcal{S}$ be the set of components of strata of $Y$ in a stratification of $f$. (Note: Smooth projective families can be regarded as proper morphisms so that $\mathcal{S}$ has only one element, the whole space $Y$.) Then according to [5] and [15], there is a (non-canonical) isomorphism in $D_{c}^{b}(Y)$ :

$$
\phi: \oplus_{i}{ }^{p} \mathcal{H}^{i}\left(f_{*} I C_{X}\right)[-i] \simeq R f_{*} I C_{X},
$$

where ${ }^{p} \mathcal{H}$ is the perverse cohomology functor 3. Moreover, for every $i,{ }^{p} \mathcal{H}^{i}\left(f_{*} I C_{X}\right)$ is semisimple; more precisely, there is a canonical isomorphism in $\operatorname{Perv}_{\mathbb{Q}}(Y)$ :

$$
{ }^{p} \mathcal{H}^{i}\left(f_{*} I C_{X}\right) \simeq \oplus_{S \in \mathcal{S}} I C_{\bar{S}}\left(\mathcal{L}_{i, S}\right)
$$

where the local systems $\mathcal{L}_{i, S}:=\mathcal{H}^{-\operatorname{dim}(S)}\left(\left.{ }^{p} \mathcal{H}^{i}\left(f_{*} I C_{X}\right)\right|_{S}\right)$ on $S$ are semisimple; these local systems underlie (a direct sum of) polarized variations of Hodge structures. In fact, all complexes appearing in (6.1) and (6.2) underlie pure Hodge modules, and both isomorphisms

\footnotetext{
${ }^{3}$ If the morphism $f$ is projective, then (6.1) is a formal consequence of the Relative Hard Lefschetz Theorem (e.g., see [36, 15]), and there is a distinguished isomorphism $\phi_{\eta}$ depending only upon the choice of a relatively ample line bundle $\eta$ on $X$ (e.g., see [16]). For more on these issues, see [10].
} 
have analogues in Saito's category of mixed Hodge modules (e.g., (6.2) follows from the decomposition by strict support of a pure Hodge module). The isomorphism (6.1) yields the degeneration at the $E_{2}$-term of the perverse Leray spectral sequence for $f$.

Remark 6.1. The isomorphisms (6.1) and (6.2) hold, with analogous interpretations at the level of mixed Hodge modules, if we replace $I C_{X}$ by a twisted intersection complex $I C_{X}(\mathbb{V})$, where $\mathbb{V}$ underlies a polarized variation of Hodge structures defined on a Zariski open dense subset of $X$.

As in Proposition 2.1 and Remark 2.3, the intersection cohomology groups of a complex algebraic variety $Z$ (with a polarized variation of Hodge structures $\mathbb{V}$ defined on a Zariskidense open subset) admit natural mixed Hodge structures, and by analogy with \$2.1 one defines Hodge-theoretic intersection homology genera $I \chi_{y}(Z)$ and respectively $I \chi_{y}(Z ; \mathbb{V})$, by encoding in a polynomial the corresponding mixed Hodge numbers. For $y=1$, these genera yield the Goresky-MacPherson signature $\sigma(Z)$ [23], and respectively the twisted signature $\sigma(Z ; \mathbb{V})$ considered in $[3]$.

In [10] the authors studied the behavior of the $I \chi_{y}$-genus under proper morphisms of projective varieties and showed that, under the trivial monodromy assumption along all the strata of $f, I \chi_{y}$ satisfies the stratified multiplicative property. Here we want to point out that the stratified multiplicative property for the $I \chi_{y}$-genus holds in the more general context of projective maps of complex algebraic varieties. This aspect will be discussed in what follows. In general, with no assumption on the monodromy along strata, one has the following result

Proposition 6.2. Let $f: X \rightarrow Y$ be a proper surjective morphism of complex algebraic varieties, and $\mathcal{S}$ be the set of components of strata of $Y$ in a stratification of $f$. Then

$$
I \chi_{y}(X)=\sum_{S \in \mathcal{S}} \sum_{i}(-1)^{n+i-\operatorname{dim}(S)} I \chi_{y}\left(\bar{S} ; \mathcal{L}_{i, S}\right) .
$$

Proof. This follows essentially from the fact that the perverse Leray spectral sequence of $f$ is a spectral sequence in the category of mixed Hodge structures (cf. \$2.3).

By definition,

$$
\begin{aligned}
I \chi_{y}(X) & :=\sum_{p} I \chi^{p}(X) \cdot(-y)^{p} \\
& :=\sum_{p, i}(-1)^{i} \operatorname{dim}_{\mathbb{C}} G r_{F}^{p} I H^{i}(X ; \mathbb{C}) \cdot(-y)^{p} \\
& =\sum_{p, i}(-1)^{i} \operatorname{dim}_{\mathbb{C}} G r_{F}^{p} \mathbb{H}^{i-n}\left(X ; I C_{X}\right) \cdot(-y)^{p} \\
& =(-1)^{n} \sum_{p} \chi^{p}\left(X ; I C_{X}\right) \cdot(-y)^{p}
\end{aligned}
$$

On the other hand, since all differentials in the perverse Leray spectral sequence for $f$ and $I C_{X}$

$$
E_{2}^{i, j}=\mathbb{H}^{i}\left(Y,{ }^{p} \mathcal{H}^{j}\left(f_{*} I C_{X}\right)\right) \Longrightarrow \mathbb{H}^{i+j}\left(X ; I C_{X}\right)=I H^{i+j+n}(X ; \mathbb{Q})
$$


are compatible with the Hodge filtrations involved, it follows that

$$
\begin{aligned}
\chi^{p}\left(X ; I C_{X}\right) & :=\sum_{k}(-1)^{k} \operatorname{dim}_{\mathbb{C}} G r_{F}^{p} \mathbb{H}^{k}\left(X ; I C_{X}\right) \\
& =\sum_{i, j}(-1)^{i+j} \operatorname{dim}_{\mathbb{C}} G r_{F}^{p} \mathbb{H}^{i}\left(Y,{ }^{p} \mathcal{H}^{j}\left(f_{*} I C_{X}\right)\right) \\
& \stackrel{(a)}{=} \sum_{i, j}(-1)^{i+j} \operatorname{dim}_{\mathbb{C}} G r_{F}^{p} \mathbb{H}^{i}\left(Y, \oplus_{S \in \mathcal{S}} I C_{\bar{S}}\left(\mathcal{L}_{j, S}\right)\right) \\
& \stackrel{(b)}{=} \sum_{i, j} \sum_{S \in \mathcal{S}}(-1)^{i+j} \operatorname{dim}_{\mathbb{C}} G r_{F}^{p} \mathbb{H}^{i}\left(\bar{S}, I C_{\bar{S}}\left(\mathcal{L}_{j, S}\right)\right) \\
& =\sum_{i, j} \sum_{S \in \mathcal{S}}(-1)^{i+j} \operatorname{dim}_{\mathbb{C}} G r_{F}^{p} I H^{i+\operatorname{dim}(S)}\left(\bar{S} ; \mathcal{L}_{j, S}\right) \\
& =\sum_{j} \sum_{S \in \mathcal{S}}(-1)^{j-\operatorname{dim}(S)} I \chi^{p}\left(\bar{S} ; \mathcal{L}_{j, S}\right) .
\end{aligned}
$$

where $(a)$ follows from the semi-simplicity part of the BBD theorem, and $(b)$ from the fact that the semi-simplicity isomorphism (6.2) induces a canonical isomorphism of mixed Hodge structures in hypercohomology. The result follows now from the first sequence of equalities above.

Remark 6.3. More generally, in the setting of Proposition 6.2 and by considering a polarized variation of Hodge structures $\mathbb{V}$ on a Zariski open dense subset of $X$, we obtain an analogous formula for the twisted genus $I \chi_{y}(X ; \mathbb{V})$ in terms of twisted genera corresponding to strata of $f$.

If $\mathbb{V}$ is a polarized variation of Hodge structures defined on a dense open Zariski subset $U$ of a variety $Z$, then under the assumption of trivial monodromy along $U$ (e.g., $\pi_{1}(U)=0$ ), we obtain as in [10] that $I \chi_{y}(Z ; \mathbb{V})=I \chi_{y}(Z) \cdot H c_{y}\left(\mathbb{V}_{x}\right)$, where $\mathbb{V}_{x}$ is the stalk of $\mathbb{V}$ at a point $x \in U$, and $H c_{y}$ is the Hodge polynomial characteristic defined by $H c_{y}\left(\mathbb{V}_{x}\right)=\sum_{p} \operatorname{dim}_{\mathbb{C}} G r_{F}^{p}\left(\mathbb{V}_{x}\right) \cdot(-y)^{p}$ (see [10], Example 3.4). Together with the (nontrivial!) identification of stalks of the local systems appearing in the BBD decomposition theorem (for details see [10]), this yields the stratified multiplicative property for the $I \chi_{y^{-}}$ genus in the more general context of projective maps of complex algebraic varieties (compare [10], Theorem 2.11, where only the special case of proper maps of projective varieties was considered):

Theorem 6.4. Let $f: X^{n} \rightarrow Y^{m}$ be a projective surjective morphism of complex algebraic varieties. Let $\mathcal{S}$ be the set of components of strata of $Y$ in a stratification of $f$, and assume $\pi_{1}(S)=0$ for all $S \in \mathcal{S}$ (or, more generally, assume trivial monodromy on open strata). For each $S \in \mathcal{S}$, define inductively

$$
\widehat{I \chi}_{y}(\bar{S})=I \chi_{y}(\bar{S})-\sum_{W<S} \widehat{I \chi}_{y}(\bar{W}) \cdot I \chi_{y}\left(c^{\circ} L_{W, S}\right),
$$


where the sum is over all $W \in \mathcal{S}$ with $\bar{W} \subset \bar{S} \backslash S$ and $c^{\circ} L_{W, S}$ denotes the open cone on the link of $W$ in $\bar{S}$. Then:

$I \chi_{y}(X)=I \chi_{y}(Y) \cdot I \chi_{y}(F)+\sum_{S \in \mathcal{S}, \operatorname{dim} S<\operatorname{dim} Y} \widehat{I \chi}_{y}(\bar{S}) \cdot\left[I \chi_{y}\left(f^{-1}\left(c^{\circ} L_{S, Y}\right)\right)-I \chi_{y}(F) I \chi_{y}\left(c^{\circ} L_{S, Y}\right)\right]$ where $F$ is the generic fiber, and $L_{S, Y}$ is the link of a stratum $S$ in $Y$.

6.2. Conjectural formulae for twisted intersection homology genera. Proposition 6.2 motivates the search for a Hodge-theoretic Atiyah-Meyer formula for twisted intersection homology genera, extending our calculation for the twisted $\chi_{y}$-genus (see Corollary 4.6) to the case of singular varieties. In order to be able to formulate an analogue of formula (4.7) in the case of singular varieties we need to define the characteristic class $\widetilde{I T_{y_{*}}}(Z)$, which is another extension to singular varieties of the Hirzebruch class (in homology) $\widetilde{T}_{y}^{*}\left(T_{Z}\right) \cap[Z]$. For simplicity, and by analogy with the case of signatures, assume $Z$ is projective.

Definition 6.5. In the notations of Definition 5.1, for an $n$-dimensional complex algebraic variety $Z$ we set

$$
\widetilde{I T_{y_{*}}}(Z):=\widetilde{M H T}_{y}\left(I C_{Z}^{H}[-n]\right),
$$

where $I C_{Z}^{H} \in M H M(Z)$ is the intersection homology complex, regarded as a pure Hodge module (cf. [36, 37, 38]). Similarly, we define twisted characteristic classes

$$
\widetilde{I T}_{y_{*}}(Z ; \mathbb{V})=\widetilde{M H T_{y}}\left(I C_{Z}^{H}(\mathbb{V})[-n]\right)
$$

for $\mathbb{V}$ a polarized variation of Hodge structures on a Zariski open dense subset of $Z$.

By [8], Lemma 3.1, it follows as in [10, Remark 3.6, that the following normalization holds: if $Z$ is smooth and pure dimensional, then $\widetilde{I T_{y_{*}}}(Z)=\widetilde{T}_{y}^{*}\left(T_{Z}\right) \cap[Z]=\widetilde{T}_{y_{*}}(Z)$.

We point out that $\widetilde{I T_{y_{*}}}(Z)$ is the un-normalized version of the characteristic class $I T_{y}(Z)$ considered in [10]. However, for a projective variety $Z$, both classes yield the same genus, $I \chi_{y}(Z)$. (Here, by genus we mean the degree of the zero-dimensional part of the above characteristic classes.) Moreover, as in [10], Theorem 3.9, the class $\widetilde{I T_{y_{*}}}$ satisfies the stratified multiplicative property for proper maps of projective varieties.

The projected formula for twisted $I \chi_{y}$-genera is contained in the following

Conjecture 6.6. Let $Z$ be a (possibly singular) normal irreducible projective variety, and $\mathbb{V}$ a polarized variation of Hodge structures defined on a dense Zariski open subset $\mathcal{U}$ on $Z$, with associated flat bundle with "Hodge" filtration $\left(\mathcal{V}, \mathcal{F}^{\bullet}\right)$. Assume that $\mathbb{V}$ extends as a polarized variation of Hodge structures to all of $Z$ (so the underlying local system is strongly transverse to $Z \backslash \mathcal{U}$, in the sense of [3] $\mathbb{A}$, and that all bundles $\mathcal{V}, \mathcal{F}^{p}$ and $G r_{\mathcal{F}}^{p} \mathcal{V}, p \in \mathbb{Z}$, extend to all of $Z$ so that if $f: X \rightarrow Z$ is any proper map with $X$ smooth and projective then $f^{*} \mathbb{V}$ is a polarized variation of Hodge structures on $X$ with Hodge bundle $\left(f^{*} \mathcal{V}, f^{*} \mathcal{F}^{\bullet}\right)$.

\footnotetext{
${ }^{4}$ If $L_{S}$ is the link in $Z$ of a stratum $S$, then since $c^{\circ} L_{S}$ is contractible, it follows that $\left.\mathbb{V}\right|_{c^{\circ} L_{S}}$ is constant, hence $\left.\mathbb{V}\right|_{L_{S}}$ is constant.
} 
Then the following Hodge-theoretic analogue of the Banagl-Cappell-Shaneson formula for the twisted signature (cf. [3]) holds:

$$
I \chi_{y}(Z, \mathbb{V})=\int_{Z} c h^{*}\left(H c_{y}(\mathcal{V})\right) \cap \widetilde{I T}_{y_{*}}(Z) .
$$

Remark 6.7. If $Z$ is smooth and pure dimensional and $\mathbb{V}$ is defined on $Z$, then the expected formula (6.8) is equivalent to (4.7).

IDEA of PROOF: Here is an outline of how to proceed in order to prove the above conjecture. For the case of twisted signatures, in [3] the authors use some bordism representability results of Siegel and Sullivan in order to pull back calculations on singular spaces to calculations on smooth spaces. As a substitute to their argument, we will pull back calculations to a resolution of singularities, then use the BBD decomposition theorem to relate the invariants on the singular variety to those of the resolution, together with an induction on the dimension of singular strata.

Let $X$ be a (projective) resolution of singularities of $Z$, and denote by $f: X \rightarrow Z$ the resolution map. We will use the same symbol $\mathbb{V}$ for the extension of polarized variation of Hodge structures to all of $Z$. Then, by assumption, its pullback $f^{*} \mathbb{V}$ is a polarized variation of Hodge structures on $X$, with associated flat bundle and Hodge filtration given by $\left(f^{*} \mathcal{V}, f^{*} \mathcal{F}^{\bullet}\right)$. The idea is to calculate the twisted $\chi_{y^{-}}$genus $\chi_{y}\left(X, f^{*} \mathbb{V}\right)$ of the resolution in two different ways: first by using formula (4.7), and second by using the BBD decomposition theorem for the push-forward of $I C_{X}\left(f^{*} \mathbb{V}\right)$.

From (4.7) we obtain the following:

$$
\begin{array}{rlrl}
\chi_{y}\left(X, f^{*} \mathbb{V}\right) & \stackrel{4.7}{=} & \int_{X}\left(c h^{*}\left(H c_{y}\left(f^{*} \mathcal{V}\right)\right) \cup \widetilde{T}_{y}^{*}(X)\right) \cap[X] \\
\qquad r_{f^{*} \mathcal{F}} f^{*} \mathcal{V}=f^{*} G r_{\mathcal{F}}^{p} \mathcal{V} & \int_{X} f^{*} c h^{*}\left(H c_{y}(\mathcal{V})\right) \cap \widetilde{T}_{y_{*}}(X) \\
= & & \int_{Z} c h^{*}\left(H c_{y}(\mathcal{V})\right) \cap f_{*} \widetilde{T}_{y_{*}}(X) .
\end{array}
$$

Since $X$ is a resolution of $Z, I C_{Z}$ is a canonical summand of $f_{*} I C_{X}$, all other terms in the $\mathrm{BBD}$ decomposition being twisted intersection cohomology complexes supported on lower dimensional strata of $Z$ (in a stratification of $f$ ). Therefore, by Definition 6.5,

$f_{*} \widetilde{T}_{y_{*}}(X)=\widetilde{I T_{y_{*}}}(Z)+$ twisted characteristic classes coming from lower dimensional strata of $Z$ Hence,

$$
\chi_{y}\left(X, f^{*} \mathbb{V}\right)=\int_{Z} c h^{*}\left(H c_{y}(\mathcal{V})\right) \cap \widetilde{I T_{y_{*}}}(Z)+\text { terms coming from lower strata of } Z .
$$

On the other hand, from the BBD decomposition theorem (cf. Remark 6.1$), I C_{Z}(\mathbb{V})$ is a canonical summand of $f_{*} I C_{X}\left(f^{*} \mathbb{V}\right)$, all other summands being supported on lower dimensional strata of $Z$. Therefore, by Remark 6.3, we obtain

$$
\chi_{y}\left(X, f^{*} \mathbb{V}\right)=I \chi_{y}(Z, \mathbb{V})+\text { terms coming from lower strata of } Z \text {. }
$$


The conjectured formula (6.8) should follow from (6.9) and (6.10), together with an induction on the dimension of strata.

We can prove the above conjecture in the following special case:

Proposition 6.8. If the variety $Z$ in the Conjecture 6.6 is assumed to have only isolated singularities, then the formula (6.8) holds.

Proof. Indeed, we first note that we can stratify the resolution map $f$ so that the singular points of $Z$ are the zero-dimensional strata, and their complement in $Z$ amounts for the top open dense stratum. Following the ideas described above, the conclusion follows if we show that, for each singular point $p \in Z$, the contribution from $p$ in the formulas (6.9) and respectively 6.10 coincide. But this follows as in 10 from the identification of the polarized (variation of) Hodge structures over $p$ in the decomposition theorem for $f_{*} I C_{X}$, and respectively $f_{*} I C_{X}(\mathbb{V})$, by using the fact that $f^{*} \mathbb{V}$ is constant on $f^{-1}(p)$, and $\mathbb{V}$ is constant on the link of $p$ in $Z$.

We conclude by pointing out that our conjecture can be also formulated for the twisted class $\widetilde{I T_{y_{*}}}(Z ; \mathbb{V})$, and we expect to follow the same lines as above for proving it.

\section{REFERENCES}

[1] Arapura, D., The Leray spectral sequence is motivic, Invent. Math. 160 (2005), 567-589.

[2] Atiyah, M.F., The signature of fiber bundles, in Global Analysis (Papers in Honor of K. Kodaira), 73-84, Univ. Tokyo Press, Tokyo, 1969.

[3] Banagl, M., Cappell, S. E., Shaneson, J. L., Computing twisted signatures and L-classes of stratified spaces, Math. Ann. 326 (2003), 589-623.

[4] Baum, P., Fulton, W., MacPherson, M., Riemann-Roch for singular varieties, Publ. Math. I.H.E.S. 45, 101-145 (1975)

[5] Beilinson, A. A., Bernstein, J., Deligne, P., Faisceaux pervers, Astérisque 100 (1982).

[6] Block, J., Weinberger, S., Higher Todd classes and holomorphic group actions, math.AG/0511305, Pure and Applied Math. Quarterly, vol. 2, no. 4, 2006 (Robert MacPherson special issue, part II).

[7] Borisov, L., Libgober, Higher elliptic genera, math.AG/0602387.

[8] Brasselet, P., Schürmann, J., Yokura, S., Hirzebruch classes and motivic Chern classes of singular spaces, math.AG/0503492.

[9] Cappell, S. E., Maxim, L. G., Shaneson, J. L., Euler characteristics of algebraic varieties, math.AT/0606654

[10] Cappell, S. E., Maxim, L. G., Shaneson, J. L., Hodge genera of algebraic varieties, I, math.AG/0606655.

[11] Cappell, S. E., Shaneson, J. L. Stratifiable maps and topological invariants, J. Amer. Math. Soc. 4 (1991), no. 3, 521-551

[12] Cappell, S. E., Shaneson, J. L., Genera of algebraic varieties and counting of lattice points, Bull. Amer. Math. Soc. (N.S.) 30 (1994), no. 1, 62-69.

[13] Carlson, J., Muller-Stach, S., Peters, C., Period mappings and Period Domains, Cambridge studies in advanced mathematics 85, 2003.

[14] Chern, S. S.; Hirzebruch, F.; Serre, J.-P., On the index of a fibered manifold, Proc. Amer. Math. Soc. 8 (1957), 587-596. 
[15] de Cataldo, M. A., Migliorini, L., The Hodge theory of algebraic maps, arXiv:math.AG/0306030 (v.1, v.2), Ann. Scient. Ec. Norm. Sup., 4e serie, t.38, 2005, p. 693-750.

[16] de Cataldo, M. A., Migliorini, L., Hodge-theoretic aspects of the Decomposition Theorem, preprint.

[17] Danilov, V. I., Khovanskii, A. G., Newton polyhedra and an algorithm for computing Hodge-Deligne numbers, Bull. AMS, Volume 30, no 1, 1994, 62-69.

[18] Deligne, P., Théorie de Hodge, II, III, Publ. Math. IHES 40, 44 (1972, 1974).

[19] Deligne, P., Equation différentielles a point singular régulier, Springer 1969.

[20] Dimca, A.; Lehrer, G. I., Purity and equivariant weight polynomials, Algebraic groups and Lie groups, 161-181, Austral. Math. Soc. Lect. Ser., 9, Cambridge Univ. Press, Cambridge, 1997.

[21] Dimca, A., Sheaves in Topology, Universitext, Springer-Verlag, 2004.

[22] Fulton, W., Introduction to toric varieties, Annals of Math. Studies 131, Princeton University Press, 1993.

[23] Goresky, M., MacPherson, M., Intersection Homology, Topology 19 (1980), 135-162.

[24] Goresky, M., MacPherson, M., Stratified Morse theory, Ergebnisse der Mathematik und ihrer Grenzgebiete (3) [Results in Mathematics and Related Areas (3)], 14. Springer-Verlag, Berlin, 1988.

[25] Griffiths, Phillip A., Periods of integrals on algebraic manifolds. I. (Construction and properties of the modular varieties.) Amer. J. Math. 901968 568-626.

[26] Hirzebruch, F., Topological methods in algebraic geometry, Springer, 1966.

[27] Hirzebruch, F., Berger, T., Jung, R., Manifolds and modular forms, Aspects of Math., vol. E20, Vieweg, 1992.

[28] Kamber, F., Tondeur, Ph., Flat manifolds, LNM no. 67, Springer, 1968.

[29] Kleiman, S., The enumerative theory of singularities, Real and Complex Singularities (P. Holm, ed.), Sijthoff and Noordhoff, 1976, pp. 298-384.

[30] Kodaira, K., A certain type of irregular algebraic surfaces, J. Analyse Math. 191967 207-215.

[31] Meyer, W., Die Signatur von lokalen Koeffizientensystemen und Faserbündeln, Bonner Mathematische Schriften 53 (Universität Bonn), 1972.

[32] Navarro Aznar, V., Sur les structures de Hodge mixtes associées aux cycles évanescents, Hodge theory (Sant Cugat, 1985), 143-153, Lecture Notes in Math., 1246, Springer, Berlin, 1987.

[33] Ochanine, S., Genres elliptiques quivariants, Elliptic curves and modular forms in algebraic topology (Princeton, NJ, 1986), 107-122, Lecture Notes in Math., 1326, Springer, Berlin, 1988.

[34] Peters, C., Steenbrink, J., Mixed Hodge structures, book in progress.

[35] Rosenberg, J., An analogue of the Novikov Conjecture in complex algebraic geometry, math.AG/0509526.

[36] Saito, M., Modules de Hodge polarisables, Publ. RIMS 24 (1988), 849-995.

[37] Saito, M., Mixed Hodge Modules, Publ. RIMS 26 (1990), 221-333.

[38] Saito, M., Introduction to mixed Hodge modules, Actes du Colloque de Théorie de Hodge (Luminy, 1987), Astérisque No. 179-180 (1989), 10, 145-162.

[39] Saito, M., Mixed Hodge complexes on algebraic varieties, Math. Ann. 316, 283-331 (2000).

[40] Schürmann, J., Topology of singular spaces and constructible sheaves, Monografie Matematyczne, 63. Birkhäuser Verlag, Basel, 2003.

[41] Schürmann, J., Yokura, S., A survey of characteristic classes of singular spaces, math.AG/0511175.

[42] Schürmann, J., private communication.

[43] Shaneson, S., Characteristic classes, lattice points and Euler-MacLaurin formulae, Proceedings ICM, Zurich, Switzerland 1994.

[44] Steenbrink, J., Intersection forms for quasihomogeneous singularities, Compositio Math. 34 (1977), 211-223.

[45] Yokura, S., A singular Riemann-Roch for Hirzebruch characteristics, Banach Center Publications, Vol. 44, Warsaw 1998. 
S. E. Cappell: Courant Institute, New York University, New York, NY-10012

E-mail address: cappell@cims.nyu.edu

A. Libgober : Department of Mathematics, University of Illinois at Chichgo, $851 \mathrm{~S}$ Morgan Street, Chicago, IL 60607, USA.

E-mail address: libgober@math.uic.edu

L. Maxim : Department of Mathematics, University of Illinois at Chicago, 851 S Morgan Street, Chicago, IL 60607, USA.

E-mail address: lmaxim@math.uic.edu

J. L. Shaneson: Department of Mathematics, University of Pennsylvania, Philadelphia, PA-19104

E-mail address: shaneson@sas. upenn.edu 